\title{
Near isogenic lines (NIL) of sorghum carrying wild type or waxy alleles of the granule-bound starch synthase (GBSS) gene have distinct effects on human gut microbiome phenotypes and host physiological characteristics
}

\section{Qinnan Yang}

Department of Food Science and Technology, University of Nebraska-Lincoln

\section{Mallory Van Haute}

Department of Food Science and Technology, University of Nebraska-Lincoln

Nate Korth

Nebraska Food for Health Center at the University of Nebraska

\section{Scott Sattler}

Wheat, Sorghum and Forage Research Unit, USDA-Agricultural Research Service, Lincoln, NE

\section{Devin Rose}

Department of Food Science and Technology, University of Nebraska-Lincoln

Anthony Juritsch

Department of Food Science and Technology, University of Nebraska-Lincoln Jing Shao

Department of Food Science and Technology, University of Nebraska-Lincoln

\section{Kristin Beede}

Department of Food Science and Technology, University of Nebraska-Lincoln

\section{Robert Schmaltz}

Department of Food Science and Technology, University of Nebraska-Lincoln

\section{Jeff Price}

Department of Food Science and Technology, University of Nebraska-Lincoln

\section{John Toy}

Wheat, Sorghum and Forage Research Unit, USDA-Agricultural Research Service, Lincoln, NE

Amanda E. Ramer-Tait

Department of Food Science and Technology, University of Nebraska-Lincoln

Andrew K. Benson ( $\nabla$ abenson1@unl.edu )

Department of Food Science and Technology, University of Nebraska-Lincoln 
Keywords: waxy starch, resistant starch, sorghum, gut microbiome, butyrate, Roseburia, Christensenellaceae

Posted Date: March 3rd, 2022

DOI: https://doi.org/10.21203/rs.3.rs-1405055/v1

License: (c) (1) This work is licensed under a Creative Commons Attribution 4.0 International License. Read Full License 


\section{Abstract}

\section{Background:}

Waxy starches contain $>90 \%$ amylopectin and are derived from grain crops carrying naturally-occurring mutations that block amylose biosynthesis. The absence of amylose in waxy starches produces unique physiochemical properties that are desirable for food processing, but the effects of increased amylopectin/amylose ratios in waxy starches on the gut microbiome and physiological characteristics of the host are not well characterized. Here, we used a whole-grain model with isogenic pairs of wild type sorghum lines and their waxy derivatives to test the hypothesis that major differences in amylose/amylopectin ratio produce significant effects on the human gut microbiome.

\section{Results:}

Fermentation of grain from waxy versus wild type derivatives produced substantial differences in overall microbiome composition, abundances of multiple taxa, and production of microbial metabolites (butyrate). Several of the taxonomic and metabolic signatures of fermentations from parental versus waxy lines were shared across fermentations with microbiomes from different human donors, including reduced levels of butyrate production and lower abundances of Roseburia and other amylolytic, butyrateproducing members of Lachnospiraceae in fermentations of waxy lines. Using a human microbiomeassociated mouse model, we also detected significant differences in microbiome composition in animals fed low-fiber diets supplemented with $20 \%$ grain from isogenic pairs of parental versus waxy derivatives of sorghum. Remarkably, these microbiome changes were accompanied by significant differences in weight gain, with animals consuming waxy sorghum gaining significantly more weight.

\section{Conclusions:}

We conclude that the benefits of waxy starches on food functionality can have trade-off effects on the gut microbiome and host physiology that could be particularly relevant in human populations consuming large amounts of waxy grains.

\section{Introduction}

Starches are large polymers of glucose joined by a-glycosidic bonds and are important components of foods and food products world-wide. Nearly all plants produce starch, which serves as a storage reserve for metabolic energy in a range of tissues [1]. In the food industry, starch is introduced into a wide array of food products, but for most applications, the physiochemical properties of starches in their native form are unsuitable [1]. These undesirable behaviors of gelling, viscosity, and stability in native starches are typically overcome by modifying the starch through chemical or physical modification, crop breeding, or a combination of the two [2]. 
Starches are made up of two major types of glucose polymers: amylose, an almost entirely linear $(1,4)$-aD-glucan, and amylopectin, a highly branched a-glucan containing both (1,4)- and (1-6)-linkages [3]. Waxy starches were discovered in the 1900s in a unique, naturally occurring variant of maize that produced starches containing almost exclusively amylopectin [4]. The absence of amylose in waxy starches produces desirable physicochemical properties (gelling, viscosity, and stability), and waxy starches are widely used by the food industry as thickeners, gelling agents, and stabilizers [5-7]. Since discovery of the waxy phenotype in maize, naturally occurring waxy variants have also been identified in many other grain crops, including sorghum, rice, proso millet, and wheat. In the cases that have been studied, causal variants of the waxy phenotypes in cereal grains are due to loss of function mutations in the gene encoding granule-bound starch synthase (GBSS), the enzyme responsible for amylose synthesis in starch granules [8-14]. Like the original waxy maize variant, starch from waxy variants of these other cereal grains contains high percentages of amylopectin and little or no amylose due to the GBSS mutation [5, 8-13].

In addition to the effects of waxy mutations on physiochemical properties of starch, the reduced amylose in waxy derivatives also has significant effects on starch digestibility. The branched structure of amylopectin increases sites for enzymatic digestion and resists the effects of retrogradation when thermally processed $[15,16]$. In sorghum, the waxy phenotype has been associated with several alleles that impair GBSS activity [17], and two loss of function alleles have been successfully introduced into elite lines (i.e., genetic backgrounds with superior agronomic traits) of grain sorghum, primarily for the purpose of enhancing fermentability for biofuel production in a crop species that can be grown readily with limited water availability [10].

In humans, the susceptibility of amylopectin to enzymatic degradation leads to nearly complete digestion of waxy starches in the upper gastrointestinal (GI) tract by host enzymes [18, 19]. Unfortunately, this increased digestibility is also associated with a higher glycemic index rating for waxy starches compared to non-waxy wild type starches $[20,21]$. The condensed straight-chain structure of amylose has fewer sites for degradation by host enzymes, and consequently, a sizable portion of ingested amylose survives transit through the small intestine and enters the colon where it serves as a substrate for fermentation by amylolytic organisms in the colonic microbiota $[22,23]$.

Resistant starches (RS), which are recalcitrant to hydrolysis and enzymatic digestion, have been shown to provide health benefits to humans, including improved glucose tolerance and cholesterol levels as well as reduced inflammatory markers and toxic biomarkers for chronic kidney disease [24-27]. Although amylose concentration influences the digestion-resistance of RS, other factors such as source, granule composition, and thermal versus chemical modification also contribute and are used to classify RS into different categories $[16,28,29]$. RS mediates metabolic improvements through microbiome-dependent and independent pathways [30-32]. The microbiome-dependent pathways appear to be mediated through RS-stimulated growth of beneficial amylolytic bacteria that metabolize RS to short chain fatty acids (SCFA) such as butyrate [33,34]. The effects of microbially-produced butyrate are pleiotropic and afford the host multiple benefits such as providing energy to colonocytes, enhancing anti-inflammatory 
functions, and protecting against allergic responses [31, 32]. Thus, in addition to promoting high glycemic responses, the absence of amylose in waxy starches presumably leads to lower levels of RS, consequently reducing RS-mediated benefits mediated by the colonic microbiota.

Although amylose has beneficial effects on both the gut microbiome and characteristics related to gut health, most of the relationships between RS and human health have been studied in the context of pure RS. We therefore know very little about the relationships of waxy starches in their native, whole-grain context with the microbiome and host health. This gap is particularly relevant in Asia and Africa, where rice and sorghum are consumed daily as whole grain staples. While studies of locally grown and consumed rice and sorghum are limited, recent work on locally grown and consumed rice cultivars in selected areas of Asia have shown that nearly half of the cultivars studied have low amylose content and would be considered waxy [35,36]. Therefore, understanding how waxy phenotypes, in the whole grain context, may influence the microbiome and host physiology are of growing importance.

To address the knowledge gaps related to effects of waxy grains on the gut microbiome in a whole-grain context, we used near-isogenic lines of wild type and waxy derivatives of sorghum to study the effects of whole-grain starch composition on the human gut microbiome. Using a combination of in vitro microbiome fermentations mouse feeding studies, we detected significant differences in the effects of waxy derivatives versus wild type parental lines on the overall composition of the microbiome and the abundances of several microbial taxa, many of which corresponded to significant decreases in abundances of beneficial microbes in treatments with waxy lines. In addition to the strong signatures of effects on the microbiome, our studies with human microbiota-associated mice also demonstrated dramatic effects of waxy starch on host weight gain, with significant increases in weight gain observed among animals fed diets with waxy versus parental wild type sorghum. Collectively, our work highlights the need to understand how traits such as waxy, which have major effects on grain composition, can have strong trade-off effects on the gut microbiome and host health characteristics.

\section{Results}

\section{In vitro fermentation of near-isogenic lines of wild type and waxy sorghum grain with human stool microbiomes yielded distinct effects on microbiome diversity}

In the first set of experiments, we examined the outcomes of in vitro microbiome fermentation reactions across a set of near isogenic lines(NILs) of sorghum derived from six different elite genetic backgrounds. Differences in amylose in the grain from each pair of NILs was estimated by measuring residual starch content after in vitro digestion (Table 1). Across the six pairs of lines used for our studies, the wild type lines yielded $3-5$ times more residual starch $(0.21 \%$ to $0.39 \%)$ than their waxy derivative $(0.07 \%$ to $0.1 \%)$. Grain from each of these six pairs of wild type lines and isogenic waxy derivatives was subsequently used in individual in vitro microbiome fermentation reactions with human stool microbiomes from 12 donors ( 3 females and 9 males) with distinct baseline microbiome compositional features (Figure S1). 
Compositional features of the microbiomes from multiple subjects showed highly significant treatment effects in fermentations of waxy compared to parental lines across microbiomes from multiple human subjects. These treatment effects (waxy versus wild type) manifested as differences in ecological metrics (a-and b-diversity) of the microbiomes as well as significant differences in the relative abundances of individual and groups of taxa. With respect to ecological metrics, the Shannon index (a-diversity) was significantly lower in fermentations with waxy sorghum when compared to wild type lines (Figure 1A). PERMANOVA of Bray-Curtis distances also showed significant differences in b-diversity $(p<0.001)$. Subsequent analysis of $\beta$-diversity in samples from each individual microbiome by canonical analysis of principal coordinates (CAP) based on Bray-Curtis distance illustrated the strength of associations between b-diversity of the microbiomes with wild type or waxy grain lines (Figures 1B and S2), and PERMANOVA of Bray Curtis distance further highlighted the statistical differences in overall microbiome composition in the microbiomes from all 12 donors ( $p<0.05$, Figure S2). When compared to fermentations of the wildtype parental lines, our results collectively show that the waxy lines had major effects on the overall a- and b-diversity of the microbiomes, and that microbiomes from each subject were able to differentiate substrates from wild type versus waxy sorghum regardless of the sorghum genetic background in which the waxy mutations were introduced.

\section{Taxonomic features of the human gut microbiome from in vitro fermentation of wild type and waxy sorghum revealed shared and individualized patterns of responsiveness among different human donors}

Given the highly significant effects of the waxy versus wild type parental grain phenotypes on both $a$ and $\beta$-diversity metrics of microbial communities, we next identified specific microbial taxa associated with the treatment effects (waxy versus wild type) from each donor microbiome. Statistical significance was tested at multiple taxonomic levels. At the phylum level, significantly higher abundances of Proteobacteria and Bacteroidetes were found in fermentation of waxy lines, whereas significantly higher abundances of Firmicutes and Actinobacteria were found in fermentations from wild type lines (Paired Wilcoxon test followed by FDR correction, Figure 2A). The trends stayed much the same at increasing levels of taxonomic resolution. At the genus level, we detected three or more genera that accounted for many of the differences at the phylum level (Paired Wilcoxon test followed by FDR correction, Figure 2B). For example, within the phylum Bacteroidetes, the genera Allistipes, Bacteriodes, Parabacteriodes and an unclassified taxon in Tannerellaceae each showed the same trend (greater abundances in fermentations of waxy lines) with statistical significance in at least three of the subject microbiomes. Similarly, Sutterella, Escherichia-Shigella, and an unclassified taxon from Enterobacteriaceae were each present at significantly higher abundances in fermentations from waxy lines from microbiomes of five or more donors. Accounting for most of the significant increase in Firmicutes from fermentations of parental lines were members of the family Lachnospiraceae, and genera from this family also showed the most consistent behavior across donor microbiomes. For example, Roseburia was significantly higher in fermentations of wild type sorghum across all 12 donor microbiomes while Blautia and Coprococcus were significantly higher in nine and ten microbiomes (Paired Wilcoxon test followed by FDR correction, Figure 2B). Independent analysis of the data by linear discriminant analysis effect size analysis (LEfSe) also identified similar bacterial taxa 
with the greatest contribution to treatment effects (waxy versus wild type; Figure 2B). LEfSe identified Escherichia-Shigella and Alistipes as the major taxonomic groups driving fermentations of waxy lines whereas the genera driving fermentations of wild type sorghum lines corresponded to members of the Lachnospiraceae, namely Roseburia, Coprococcus, and Blautia.

\section{Individual species of Roseburia are highly responsive to amylose content across different human donors}

The most consistent microbiome-wide treatment effect (waxy versus wild type) across hosts corresponded to increased abundances of the amylolytic genus Roseburia in fermentations of grain from parental versus waxy lines. Using species-specific qPCR reactions, we confirmed the observations from the 16S rRNA sequencing data and found that this behavior was shared by three of the major Roseburia species from human microbiomes ( $R$. intestinalis, $R$. hominis, and $R$. inulinivorans) as each of these species were significantly enriched in fermentations from wild type parental sorghum lines across microbiomes from ten or more subjects compared to fermentations from near-isogenic waxy derivative lines (Figure $3 \mathrm{~A}$ and B). R. faecis was much less enriched, showing significant differences in only three out of twelve subjects (Figure $3 A$ and $B$ ).

Comparisons of CAZyme glycohydrolase families $(\mathrm{GH})$ found in the genomes of representative strains of these four human Roseburia species offers an explanation as to why $R$. intestinalis, $R$. hominis, and $R$. inulinovorans were more responsive to differences in amylose content compared to $R$. faecis. Genomes of $R$. intestinalis, $R$. hominis and $R$. inulinivorans had five to fourteen different genes encoding GH3family enzymes associated with starch degradation whereas $R$. faecis carried only two genes encoding $\mathrm{GH} 3$ enzymes (Figure 3C). Thus, enrichment of the GH3 enzyme family in $R$. intestinalis, $R$. hominis, and $R$. inulinivoransmay provide a selective advantage for growth on starch-rich substrates and may explain why these three species were more responsive to treatment effects (waxy versus wild type) across individual microbiomes.

\section{Waxy sorghum leads to reduced butyrate production in in vitro fermentations with microbiomes from multiple human donors}

The significant decreases in the abundances of amylolytic, butyrate-producing members of the Lachnospiraceae (e.g., Roseburia) in fermentations of waxy lines across multiple microbiomes would also be expected to be accompanied by decreased production of butyrate, the major end-product of starch fermentation by these organisms. Measurement of the major SCFAs by gas chromatography of supernatants from the fermentations (Figure 4A) confirmed this hypothesis, with significantly lower concentrations of butyrate in fermentations from waxy lines compared to wild type (average of $24 \%$ decrease, $p<0.001$, rANOVA followed by FDR correction). Concentrations of the other major SCFA (acetate, propionate, isobutyrate and isovalerate) were not significantly affected by treatment (waxy versus wild type lines).

Correlation analysis (Spearman's correlation) further supports substantial roles for members of the Lachnospiraceae in butyrate production as the relative abundances of Roseburia(9 out of 12 
microbiomes) and Coprococcus (8 out of 12 microbiomes) had some of the strongest correlations with butyrate production (Figure 4B, Spearman's correlation with FDR correction). Notably, Butyricicoccus, Blautia,and Faecalibacterium also showed significant correlations with butyrate production in at least 5 different microbiomes (Figure 4B). Thus, while butyrate production in these fermentations is polymicrobial, members of the Lachnospiraceae family, particularly Roseburia and Coprococcus, seem to have the most significant microbiome-wide contributions to butyrate production and these taxa are known to possess pathways for fermentation of glucose to butyrate [37], thus suggesting that they are efficient at utilizing amylose present in the wildtype parental sorghum lines.

\section{Resistant starch extracted from wild type sorghum lines restored amylose deficiency in waxy sorghum}

Mutations affecting the synthesis of major seed components (i.e., starches in the endosperm) can also have pleiotropic effects on other major components of the seed (i.e., protein content) [38]. We therefore used "molecular complementation" experiments to confirm that the differential microbiome effects observed in fermentation of whole grain from wild type and waxy lines were due to amylose content alone and not an unknown pleiotropic effect of the GBSS mutations on other seed components. Molecular complementation was achieved by introducing residual, digestion-resistant starch purified from wild type sorghum lines into fermentations with grain from amylose-deficient waxy lines and examining effects on microbiome phenotypes. Microbiome data from the fermentations were analyzed by comparing Bray-Curtis distance of microbiomes from i) fermentation of waxy sorghum lines alone, ii) fermentations of waxy lines supplemented with amylose-enriched (digestion-resistant) starch from wildtype lines, and iii) fermentations of wild type lines alone. The addition of the residual, digestionresistant starch from wild type lines indeed caused significant shifts in b-diversity in the microbiomes across multiple human subjects, with microbiomes from most subjects responding to complementation with profiles that were intermediate to profiles from fermentation of parental or waxy lines (Figures 5 and S3) but demonstrating statistically significant responses to complementation (Kruskal-Wallis test followed by post hoc pairwise multiple comparisons using Dunn's Test; Figure 5A).

We further investigated molecular complementation at higher taxonomic resolution by qPCR quantification of Roseburia species in the fermentation reactions (Figure $5 \mathrm{C}$ ). These reactions showed significant increases in the abundance of $R$. intestinalis, $R$. hominis, and $R$. inulinivorans but not $R$. faecis in the fermentations from waxy lines complemented with resistant starch from wild type lines compared to fermentations from waxy sorghum lines (Kruskal-Wallis test followed by post hoc pairwise multiple comparisons using Dunn's Test, Figure 5C). Molecular complementation of residual starch from digestion into fermentations with waxy sorghum lines also produced an expected stimulation in butyrate production, similar to the levels observed during fermentations with wild type lines (rANOVA followed by FDR correction, Figure 5D). Thus, introduction of amylose-enriched, digestion-resistant starch from parental lines into fermentation reactions of waxy lines promotes restoration of microbiome profiles in fermentations from waxy lines to profiles that are observed from fermentations of wild type parental sorghum lines, including stimulation of some of the most responsive amylolytic taxa (Roseburia) and concomitant changes in butyrate production. Consequently, the significant microbiome phenotypes 
caused by the waxy mutation in our in vitro fermentation reactions appear to be primarily dependent on the effects of waxy mutations on amylose content of the grain.

\section{Waxy behaves as a Microbiome-Active Trait in many small grain commodities}

The waxy starch trait has been developed in many small grain plant species due to its unique physicochemical properties. As with our sorghum lines, waxy lines of these other small grain species have a lower concentration of digestion-resistant starch compared to corresponding wild type lines after digestion (Table 1). To determine if the waxy trait in a whole-grain context from other species of small grains shows similar effects on the microbiome as we observed in sorghum, we compared in vitro fecal fermentations on grain derived from waxy and wild type lines of sorghum, maize, millet, rice, and wheat using the same 12 donor microbiomes for all grains tested. While the sorghum and maize grain for this experiment were derived from NILs of parental and waxy derivatives, grain from wheat, rice, and millet were not derived from isogenic pairs.

Combined data from all 12 human microbiomes showed significant reduction of butyrate production in fermentations of waxy grain from rice, sorghum, and maize with the most significant reduction occurring between waxy and wild type lines of sorghum (Paired Wilcoxon test, Figure 6A). Microbiome analysis revealed significant abundance differences in many genera (Figure 6B) and, like the butyrate production data, microbiome responsiveness to waxy and wild type lines of sorghum showed the most significant taxonomic responses based on the number of taxa showing statistically significant responses. A small number of microbial taxa showed shared responses to wild type versus waxy fermentations across multiple crop species (e.g., waxy grain from sorghum, maize, and millet all yielded significant reductions in abundances of Roseburia and elevated abundances of Escherichia based on two-way rANOVA with FDR correction). However, the taxonomic responses were largely unique to each of the crop species (Figure 6B). The unique effects of waxy wheat and rice may be due in part to the lines not being isogenic (e.g., contribution from variation at other genetic loci) and/or differences in the penetrance of the waxy mutations or differences in the physiochemical characteristics of amylose from those species (e.g., different degrees of polymerization). The important finding, however, is that human microbiomes appear to display significantly different fermentation patterns of grain from waxy versus wild type lines from each of the crop species and further experimentation is clearly warranted to understand relationships between waxy phenotypes in these crop species, physiochemical characteristics of the starches, and their impacts on fermentation by gut microbes.

\section{The waxy phenotype in whole grain sorghum alters the gut microbiome in human microbiome-associated mice}

While in vitro fermentation with human microbiomes is an excellent model for estimating the capacity of different substrates to influence the microbiome, little is known about how substrate-driven microbiome changes under in vitro growth conditions relate to the capacity of the same substrates to drive changes in the microbiome in humans or animal model systems. To address this knowledge gap, we used a human microbiota-associated (HMA) mouse model to determine if significant microbiome changes could be 
detected in HMA lines fed diets supplemented with $20 \%$ whole grain flour from isogenic wild type or waxy sorghum lines. Four unique HMA mouse lines were created by colonizing germ-free C57BL/ 6 mice with one of four human microbiomes (S766, S772, S776, and S778) demonstrating the most significant differential responses to the sorghum substrates during in vitro fermentation studies (Figure 7A). After introduction of the microbiomes, the HMA lines were divided into three treatment groups of six animals per treatment per HMA line. Among the treatments within an HMA line, one was fed a low-fiber diet while the others were fed diets supplemented with $20 \%$ sorghum from either the wild type line or the isogenic waxy derivative.

Characterization of fecal (eight time points) and cecal (terminal time point) bacterial communities of HMA mice by $16 \mathrm{~S}$ rRNA gene sequencing over time revealed that both wild type and waxy sorghum diets increased the a-diversity (Shannon index, total ASVs, and Pielou's evenness index) of the bacterial community compared to a low fiber control diet. There were no significant differences in the Shannon index or number of ASVs in the microbiomes of mice fed waxy or wild type sorghum diets, but a lower Pielou's evenness index was observed in mice fed waxy sorghum (Kruskal-Wallis test followed by post hoc pairwise multiple comparisons using Dunn's Test, Figure 7B). In contrast, constrained ordination analysis of Bray-Curtis distances showed significant differences $(p<0.05$, PERMANOVA) in the b-diversity of HMA mouse microbiomes from all four human donors when fed a waxy sorghum diet compared to a wild type sorghum diet (Figure $7 \mathrm{C}$ ). Thus, even with sorghum representing only $20 \%$ of the diet, significant treatment effects (wild type versus waxy sorghum) were detected in the microbiomes of all four HMA mouse lines.

Consistent with the changes in $\beta$-diversity, significant differences in the relative abundances of bacterial genera were also detected in each HMA mouse line fed diets containing waxy versus wild type sorghum (Paired Wilcoxon test followed by FDR correction, Figure 7E). The patterns of taxa showing statisticallysignificant treatment effects (waxy versus parental) were unique to each individual microbiome, a result commonly observed in experiments using HMA mice that harbor different donor microbiomes [39-41]. In HMA mice carrying the stool microbiome of subject S772, taxa that were significantly less abundant in animals fed the waxy sorghum diet included members of the Bacteriodales (Muribaculaceae), Erysipelotrichiaceae (Allobaculum), Lachnospiraceae (Agathobacter and Anaerostipes), Ruminococcaceae (Faecalibacterium), Clostridia (Christensensellaceae) and Enterococcaceae (Enterococcus) whereas abundances of Erysipeloatoclostridium (Erysipelotrichaceae) and Phascolarctobacterium (Acidaminococcaceae) were higher in mice fed waxy sorghum. Microbiomes from HMA mice harboring stool from subject S778 shared some overlapping taxonomic responses with those carrying the $\mathbf{S 7 7 2}$ microbiota when receiving waxy sorghum compared to wild type sorghum, including decreased abundances of Christensenellaceae and increased abundances of Erysipeloatoclostridium and Phascolarctobacterium. Another intriguing aspect of the microbiomedependent responses was the observation that treatment effects on several of the significant taxa were not necessarily in the same direction for each microbiome, implying that the microbiome context is a major determinant of how individual microbial taxa may respond. However, we did note that three taxa, Faecalibacterium, Christensenellaceae, and Enterococcus, exhibited significant diet-driven responses in 
the same direction (e.g., decreased in waxy sorghum diet) across HMA mouse lines from two or more donor microbiomes.

Although dietary treatments (wild type versus waxy) had significant effects on compositional features of the microbiomes in HMA mouse lines, a notable difference between responses of the microbiomes in the in vitro fermentations compared to the HMA feeding experiment was the absence of treatment effects on Roseburia in the HMA mice.Given the strong responsiveness of this organism to wild type versus waxy grain in the in vitro experiments and the consistency of this response across multiple human microbiomes (Figure 2), absence of significant treatment effects in the HMA mice was unexpected. These disparate results are explained, however, by inefficient colonization of Roseburia species, as qPCR assays for Roseburia species (Figure S4) showed that populations of Roseburia species declined rapidly after microbiome introduction in all four HMA lines. Indeed, by day 7, populations of all four Roseburia species declined to levels near the threshold for detection, suggesting that Roseburia did not efficiently engraft and persist in ex-germ-free mice. Further study is needed to determine if poor colonization of the mouse host is a general feature of Roseburia.

In addition to the microbiome phenotypes, we also tested for treatment effects (wild type versus waxy) on feed intake and weight gain throughout the study. Remarkably, we found that animals from all four HMA mouse lines fed diets supplemented with waxy sorghum gained significantly more weight compared to their counterparts receiving either the wild type sorghum or low fiber control diets (rANOVA followed by FDR correction, Figure 7D). These weight gain phenotypes were observed in the absence of any significant treatment effects on feed intake, and are thus driven by compositional differences (e.g., amylose content) between the diets alone, most likely by more efficient digestion of the higher levels of amylopectin in feed derived from waxy lines. Studies comparing weight gain from consumption of wild type and waxy grains are limited, but one study in broilers demonstrated significant increases in weight gain in animals fed grain from waxy versus non-waxy hybrids of maize [42].

\section{Discussion}

Although waxy grains and starches are widely consumed [5, 43-45], little is known about how these starches affect the gut microbiome or other characteristics related to human health. To begin filling this knowledge gap, we used a combination of in vitro fermentation and pre-clinical animal models to assess effects of whole grain from parental lines versus their cognate waxy derivatives on compositional and metabolic features of microbiomes from stool samples of multiple human donors. For this study, we capitalized on powerful plant resource populations comprising multiple pairs of NILs in which the waxy trait was bred into different elite parental backgrounds, thus providing biological replication of the waxy trait across multiple genetic backgrounds. Grains from these lines was used for both in vitro and in vivo studies to examine the influence of starch composition on the human gut microbiome.

\section{In vitro fermentations using microbiomes of multiple human donors demonstrate that the waxy phenotype in sorghum is an example of a Microbiome-Active Trait.}


Results from our in vitro fermentation study revealed significant differences in the effects of waxy versus wild type grain (treatment effects) on ecological metrics (a- and b-diversity), taxonomic features, and metabolite profiles. An unexpected finding from the in vitro fermentation studies was the consistency of treatment effects on butyrate production and abundances of specific amylolytic, butyrate-producing taxa that were detected across diverse microbiomes of the different human donors. Different genera of the family Lachnospiraceae were consistently less abundant in fermentations with amylose-deficient waxy sorghum. This conserved microbiome signature effect was particularly strong for Roseburia, which was significantly reduced in fermentations of waxy grain from all 12 of the donor microbiomes tested. This finding is of particular interest because increased abundances of Roseburia have been associated with reduced susceptibility to inflammatory and metabolic diseases [46-51]. Roseburia species are known to degrade starch [52] and produce butyrate as a primary end-product of fermentation [53]. Moreover, our molecular compelementation studies confirmed that the amylose-enriched RS purified from wild type lines alone could complement the defects of waxy grain in promting growth of Roseburia and butyrate production. We also highlight a number of different human feeding studies where dietary supplementation with RS was associated with increases in Roseburia in the gut microbiome [40,48, 54, 55]. Thus, despite the differences in ecological environments of in vitro fermentations and human feeding studies, the in vitro fermentation model may be quite relevant for mechanistic studies of Roseburia species in humans.

\section{The waxy trait in sorghum behaves as a Microbiome-Active Trait in human microbiome-associated mice}

Human microbiota-associated (HMA) mice (germ-free mice inoculated with a donor human gut microbiome) are powerful tools for mechanistic study of human microbiomes [56-58]. In our experiments, HMA mice were fed whole-grain sorghum supplemented diets to determine if the significant treatment effect (waxy versus wild type) on the gut microbiome observed in vitro could be recapitulated in HMA lines carrying the same microbiomes used for in vitro experiments. An important consideration for these experiments was that the diet fabrication process limited the amount of whole grain sorghum that could be introduced to $20 \%$ whole grain sorghum from the isogenic pair of wild type/waxy-derivative lines. This amount equates to only a modest difference in amylose content between the two diets of approximately $2 \%$ (in the wild type line), whereas studies with purified substrates typically use higher concentrations of RS or other fibers (e.g., 10\% to 30\%) [30,59]. Nonetheless, even with this modest difference in dietary amylose content, we detected significant treatment effects of diet (waxy versus wild type) on overall compositional features of the microbiome (e.g., b-diversity) as well as changes to several individual taxa. Treatment effects were significant across all four HMA mouse lines, but the differences in taxonomic configurations were unique to a given donor microbiome, a result that is often observed in experiments with HMA mice [39, 40].

Perhaps the most intriguing outcomes from the feeding experiments in HMA lines were that i) some taxonomic differences between waxy and wild type sorghum diets were shared across HMA mouse lines from more than one donor and that ii) animals fed waxy diets had statistically significant increases in weight gain, regardless of the donor microbiome. With respect to taxonomic effects, genera such as 
Muribaculaceae, Anaerostipes, Eubacterium, Agathobacter, Blautia, Erysipelatoclostridium, and Phascolarctobacterium showed significant effects of treatment (diet) in two or more of the HMA mouse lines, but the directionality of the effects for these taxa were not necessarily consistent across lines, suggesting their responsiveness is dependent on context of the microbiome. In contrast, the genus Faecalibacterium and members of the taxonomic group Christensenellaceae $R$ - 7 group showed consistent effects in the same direction, with significantly lower in abundances in animals fed amylosedepleted waxy sorghum diets in two or more of the HMA mouse lines. Both organisms are well-regarded as beneficial microbes. For example, abundances of Christensenellaceae are associatedwith longevity [60,61], reduced susceptibility to inflammatory bowel diseases [62], and positive effects on body mass index [63-65] while the genus Faecalibacterium is associated with human health benefits and negatively correlates to inflammatory bowel disease [66-69].

The microbial taxa showing significant responses to diet in the HMA mice were largely distinct from the taxa affected in vitro. This result is not surprising since the in vitro fermentation conditions are quite different from the mouse gut ecosystem. In particular, Roseburia failed to show significant responses to diet in the HMA lines, despite their strong responsiveness in in vitro fermentations. This outcome may be due to challenges with getting Roseburia species from the human microbiomes to efficiently colonize the mouse gut in our HMA model. Attempts to quantify Roseburia species in the HMA lines with our speciesspecific qPCR assays confirm this hypothesis, with most species colonizing at levels $<10^{5} \mathrm{CFU} / \mathrm{g}$ of feces and some declining to even lower levels over time (Supplemental Figure 4). In contrast to the situation with Roseburia, other families containing prominent amylolytic members such as genera Faecalibacterium from the Ruminococcaceae family showed consistent and significant responses to the dietary treatments in the HMA mouse lines from multiple microbiomes (Figure 2 and Figure 7). In this instance, it may be that composition of the media for our in vitro fermentations favors growth of members of the Lachnospiraceae. While we absolutely expect there to be differences in responsiveness of organisms under in vitro conditions versus in vivo in HMA mouse lines, the focus of our study and the more relevant outcome is that both in vitro and in vivo experimental approaches showed that grain from wild type and waxy derivatives causes significant differences in the microbiome, leading us to conclude that the waxy phenotype in sorghum behaves as a Microbiome-Active Trait.

\section{The waxy phenotype in small grains may have potentially undesirable effects on human health, particularly in populations consuming high levels of waxy grains as a staple}

Waxy starch traits have been identified and/or introduced by conventional breeding in many small grain crop species [8-13], and these traits also occur naturally in locally cultivated and consumed varieties of rice that are a staple food in some geographies [20]. In the instances where it has been measured, waxy grains have higher ratings on the glycemic index (i.e., propensity for increasing blood glucose levels) than non-waxy, largely due to the higher levels of readily digestible amylopectin in waxy lines [20,21]. Thus, there could be significant, long-term implications for health outcomes in populations consuming waxy grain on a regular basis. In addition to the potential physiological consequences of diets containing grains that rate high on the glycemic index, our data supports the conclusion of the waxy trait also 
behaving as a Microbiome-Active Trait with undesirable effects on the microbiome. The waxy starch trait can be considered as a Microbiome-Active Trait because the absence or decreased levels of amylose in waxy lines reduces RS content and the beneficial effects of RS on the gut microbiome, thus essentially decreasing a significant fiber benefit of the grain. Recent studies of the glycemic index for a broad panel of rice cultivars, including improved cultivated varieties, showed a strong association of naturallyoccurring waxy alleles with elevated glycemic index values and a significant negative correlation between amylose content and glycemic index rating [20]. Studies have also showed significant associations between amylose content, predicted glycemic index and glycemic load, and allelic variation linked to waxy variants $[35,36]$. More detailed studies of health outcomes and microbiome composition in populations consuming large amounts of waxy or high-amylose rice could therefore illuminate the potential for waxy rice consumption to predispose the local populations to type II diabetes [21, 36]. More research needs to be done to determine the negative ramifications of consuming high quantities of waxy grain.

With respect to crop improvement, there is growing recognition that traits such as glycemic index should be included as targets for improvement, and high-throughput methods for phenotyping glycemic index have been reported [70]. Given our results and the known beneficial effects of RS on the human gut microbiome and human health, we further posit that high-throughput methods for microbiome phenotyping, such as in vitro fermentation, can also be incorporated into improvement programs, permitting improvement of crops for Microbiome-Active Traits that may also be associated with human health.

\section{Conclusions}

The findings of this study revealed that the wide use of waxy starches in food products could potentially have significant undesirable effects on the composition and function of the gut microbiome compared to foods with native, amylose-containing starches. Although our study does not permit direct inference of the effects of waxy as a Microbiome-Active Trait on human health, the organisms most affected by waxy grain in in vitro fermentations (e.g., Roseburia) and the HMA mouse model (e.g., Faecalibacterium) are well regarded as beneficial microbes. These undesirable microbiome effects of waxy grain were also accompanied by a weight gain phenotype in HMA mice. Thus, it is reasonable to hypothesize that longterm consumption of waxy grains may have multiple unintended and undesired effects that are relevant to health. Our work illuminates the need to better understand the potential trade-offs between functional traits and Microbiome-Active Trait, and for plant breeders and food scientists to consider these trade-offs when developing hybrids for food ingredients and food product formulations.

\section{Materials And Methods}

\section{Grain information}


The information of the grains used in this study are provided in Table 1. Six waxy grain sorghum lines and their near iso-genic wild type lines were obtained from the U.S. Department of Agriculture, Agricultural Research Service (USDA-ARS), Wheat, Sorghum and Forage Research Unit (Lincoln, NE). The amylose content in sorghum were determined using a commercial kit according to the manufacturer's instructions (K-AMYL Amylose/Amylopectin Assay Kit, Megazyme, Wicklow, Ireland).

\section{In vitro digestion}

Whole grain samples were digested following established procedures [71]. Briefly, $5 \mathrm{~g}$ of whole grain were ground into fine powder using Geno/Grinder 2025 (SPEX SamplePrep) at $1600 \mathrm{rpm}$ for $10 \mathrm{~min}$. Then, 2.5 $\mathrm{g}$ of sample was mixed with $30 \mathrm{~mL}$ of water in a $50 \mathrm{~mL}$ Falcon tube for 20 min until fully dispersed. Tubes containing the slurries were immersed in boiling water for 20 min with constant shaking. The slurries were then placed on an orbital shaker $(200 \mathrm{rpm})$ and incubated at $37^{\circ} \mathrm{C}$ for $40 \mathrm{~min}$. The pH was adjusted to 2.5 with $1 \mathrm{M} \mathrm{HCl}$ followed by the addition of $1 \mathrm{~mL}$ of $10 \%$ (wt/vol) pepsin (P7000; Sigma, St. Louis, MO) in $50 \mathrm{mM} \mathrm{HCl}$. The slurry was then incubated on an orbital shaker (200 rpm) at $37^{\circ} \mathrm{C}$ for 60 min. The $\mathrm{pH}$ was adjusted to 6.9 with $0.5 \mathrm{M} \mathrm{NaHCO}_{3}$ and $5 \mathrm{~mL}$ of $12.5 \%$ (wt/vol) pancreatin (P7545; Sigma, St. Louis, MO) in $0.1 \mathrm{M}$ sodium maleate buffer, and $0.2 \mathrm{~mL}$ of amyloglucosidase (E-AMGDF, 3,260 $\mathrm{U} / \mathrm{mL}$, Megazyme) was added. The slurry was incubated for $6 \mathrm{~h}$ at $37^{\circ} \mathrm{C}$ with orbital shaking at $200 \mathrm{rpm}$. Following digestion, the material was transferred to dialysis tubing (molecular weight cutoff of 2000 Dalton) and dialyzed against distilled water for 3 days at $4^{\circ} \mathrm{C}$ with a water change every $12 \mathrm{~h}$. The retentate from the dialysis was collected, freeze-dried and resuspended in $30 \mathrm{~mL}$ of sterile distilled water. The residual starch concentration was determined with a commercial kit using the protocol variation entitled "Determination of total starch content of samples containing resistant starch" (K-TSTA, Total Starch Assay Kit (AA/AMG), Megazyme).

\section{Fecal donor and in vitro fecal fermentation}

Fresh fecal samples from 12 healthy adults with no history of gastrointestinal abnormalities and no prebiotic, probiotic, or antibiotic consumption within the past six months were collected using a commode specimen collection kit (Fisher Scientific, NH, USA). All procedures involving human subjects were approved by the Institutional Review Board of the University of Nebraska-Lincoln before initiating the study (20160816311EP). A 1:10 fecal slurry was prepared in an anaerobic chamber $(5 \% \mathrm{H} 2,5 \% \mathrm{CO}$, and $90 \% \mathrm{~N} 2$; Bactron X, Sheldon Manufacturing, Cornelius, OR, USA) within $2 \mathrm{~h}$ of collection by adding sterile $10 \%$ glycerol in phosphate-buffered saline, $\mathrm{pH} 7.0(1: 9, \mathrm{w} / \mathrm{v})$ and mixing with a stomacher for $4 \mathrm{~min}$ prior to storing at $-80^{\circ} \mathrm{C}$ until fermentation.

In vitro batch fermentations were performed inside an anaerobic chamber. Two hundred fifty microliters of resuspended sample was mixed in a $1 \mathrm{~mL}$-deep well in a 96-well plate with $0.25 \mathrm{~mL}$ of $2 \mathrm{X}$ fermentation medium containing (per liter): $1 \mathrm{~g}$ Bacto casitone, 1 g yeast extract, $2 \mathrm{~g} \mathrm{~K} 2 \mathrm{HPO} 4,3.2 \mathrm{~g} \mathrm{NaHCO} 3,3.5 \mathrm{~g}$ $\mathrm{NaCl}, 1 \mathrm{~mL}$ hemin solution ( $\mathrm{KOH} 0.28 \mathrm{~g}, 95 \%$ Ethanol $25 \mathrm{~mL}$, hemin $100 \mathrm{mg}$ and ddH2O to $100 \mathrm{~mL}$ ), 0.05 $\mathrm{g}$ bile salts, $0.5 \mathrm{~g} / \mathrm{L}$ cysteine $\mathrm{HCl}, 0.6 \mathrm{~mL}$ resazurin (0.1\%), $10 \mathrm{~mL}$ ATCC trace mineral supplement, $3.6 \mathrm{~mL}$ 
VFA solution (17 mL acetic acid, $1 \mathrm{~mL}$-valeric acid, $1 \mathrm{~mL}$ iso-valeric acid, $1 \mathrm{~mL}$ iso- butyric acid mixed with $20 \mathrm{~mL}$ of $10 \mathrm{mM} \mathrm{NaOH}), 10 \mathrm{~mL}$ ATCC vitamin supplement and $1 \mathrm{~mL}$ vitamin $\mathrm{K}-3$ solution $(0.14 \mathrm{~g}$ vitamin $\mathrm{K}-3$ in $100 \mathrm{~mL}$ 95\% ethanol) [72]. The substrate was then reduced at $4^{\circ} \mathrm{C}$ in the anaerobic box with anerobic gas generator (Mitsubishi ${ }^{\mathrm{TM}}$ AnaeroPack-Anaero, Japan) for 3 days before inoculation with $0.05 \mathrm{~mL}$ of fecal slurry. In vitro fermentations were incubated at $37^{\circ} \mathrm{C}$ for $16 \mathrm{~h}[73,74]$. After fermentation, samples were centrifuged at $4000 \mathrm{~g}$ for $10 \mathrm{~min}$. Pellets and supernatants were stored at $-80^{\circ} \mathrm{C}$ until further processing.

\section{Starch complementation experiment}

Starch from wild type lines 'Wheatland' and 'Tx430' was extracted using a modified protocol from Xie et al. [75]. The isolated starch then underwent in vitro digestion as described previously [71]. The resulting starch concentration was determined as described above. The digested starch was added to digested waxy sorghum substrate (starch concentration: waxy Wheatland, $2.79 \mathrm{mg} / 100 \mathrm{~mL}$; waxy Tx430, 2.24 $\mathrm{mg} / 100 \mathrm{~mL}$ ) to adjust the starch concentration to that of the corresponding wild type sorghum substrate (starch concentration: wild type Wheatland, $12.82 \mathrm{mg} / 100 \mathrm{~mL}$; wild type Tx430, 9.85 mg/100 mL), thus making the starch concentration $9.31 \mathrm{mg} / 100 \mathrm{~mL}$ for supplemented waxy Wheatland and $6.57 \mathrm{mg} / 100$ $\mathrm{mL}$ for supplemented waxy Tx430. The digested starch was also added to wild type sorghum (starch concentration: supplemented wild type Wheatland, $20.22 \mathrm{mg} / 100 \mathrm{~mL}$; supplemented wild type Tx430, $13.27 \mathrm{mg} / 100 \mathrm{~mL}$ ) to further test the dose response of microbiomes to starch concentration. Waxy sorghum, waxy sorghum supplemented with starch, wild type sorghum, and wild type sorghum supplemented with starch were each inoculated into one of six human fecal microbiomes with the largest and smallest differences between waxy and wild type sorghum observed during in vitro fermentations (largest and smallest $\mathrm{R}^{2}$ value from PERMANOVA analysis, data not shown) as described above.

\section{Human microbiota-associated mice}

Female germ-free C57BL/6 mice were born and reared in flexible film isolators and maintained under gnotobiotic conditions (temperature $20^{\circ} \mathrm{C}$, relative humidity $60 \%, 14 \mathrm{~h}$ light/10 h dark cycle) at the University of Nebraska-Lincoln. Germ-free status of the breeding colony was checked routinely as described [30]. The Institutional Animal Care and Use Committee at the University of Nebraska-Lincoln approved all procedures involving animals (protocol 1700). At 12-13 weeks of age, germ-free mice were transferred from isolators to autoclaved individually ventilated cages mounted on racks with positive airflow as previously described [30] and then colonized immediately with human stool microbiomes. Four human fecal microbiomes exhibiting the greatest differences between waxy and wild type sorghum during in vitro fermentations (largest $\mathrm{R}^{2}$ value and smallest $\mathrm{P}$ value from PERMANOVA analysis, data not shown) were selected for inoculation into mice. To establish human microbiome-associated (HMA) mice, $100 \mathrm{uL}$ of each human fecal slurry ( $N=18$ mice for each donor) was orally gavaged into mice once. Mice were then randomly assigned to dietary treatments based on body weight at the time of colonization and housed three per cage by donor microbiome. No significant differences in body weight were observed among treatment groups on the day of randomization (data not shown). Fecal pellets were collected from 
individual mice twice a week and stored at $-80^{\circ} \mathrm{C}$ until DNA extraction. Cecal contents were collected at necropsy and stored at $-80^{\circ} \mathrm{C}$ until DNA extractions were performed as previously described [76].

\section{Experimental diets}

Mice were fed an autoclaved chow diet (LabDiet 5K67, Purina Foods, St. Louis, MO) ad libitum prior to the introduction of experimental diets at the time of colonization with human stool microbiomes.

Experimental diets were prepared and irradiated by Research Diets, Inc. (New Brunswick, NJ). Mice were fed either a low fiber control diet based on the AIN-93G rodent diet formulation with dextrose as the sole carbohydrate source or a customized diet based on AIN-93G where the dextrose was replaced with $20 \%$ Wheatland sorghum flour from either wild type or waxy isogenic line. Six mice for each donor microbiome and diet combination were fed for four weeks. Diet compositions are listed in Supplementary Table 1.

\section{DNA extraction and 16S rRNA gene sequencing}

DNA was extracted from mouse fecal pellets and cecal contents using the BioSprint 96 workstation (Qiagen, Germantown, MD) and the BioSprint 96 one-for-all Vet kit with the addition of buffer ASL (Qiagen) and bead beating [77]. The V4 region of the bacterial 16S rRNA gene was amplified from each sample using the dual-indexing sequencing strategy [78].

\section{S rRNA gene sequencing processing}

Paired-end sequences were analyzed using Quantitative Insights Into Microbial Ecology (QIIME) program (version 2) [79]. Sequences were truncated (220 bases for forward reads and 160 bases for reverse reads) and denoised into amplicon sequence variants (ASVs) using DADA2 [80]. All ASVs were assigned with taxonomic information using pre-fitted sklearn-based taxonomy classifier SILVA database (release 138) [81] and were then binned at genus level and transformed to relative abundance by dividing each value in a sample by the total reads in that sample. PICRUSt2 was used to generate predicted metagenomes [82].

\section{Investigation of Roseburia}

To quantify individual Roseburia species responses to waxy sorghum, we used qPCR to identify four Roseburia species. Representative genomes of type strains Roseburia intestinalis DSM 14610, Roseburia inulinivorans DSM 16841, Roseburia hominis DSM 16839 and Roseburia faecis DSM 16840 were downloaded from the NCBI database (http://www.ncbi.nlm.nih.gov/genome) and analyzed using dbCAN2 [83] to investigate the functions of carbohydrate utilization. Species-specific primers were designed using Rapid identification of PCR primers for unique core sequences (RUCS) [84]. Primer specificity was validated by Primer BLAST against the NCBI RefSeq representative genome database [85]. The validated species-specific primer sequences used were: Roseburia intestinalis FCGAAGCACTTTATTTGATTTCTTCGG, R- TTTTTCACACCAGGTCATGCG; Roseburia hominis FAAGTCTTGACATCCCACTGACA, R- CACCACTGCTCCGAAGAGAA; Roseburia inulinivorans FGACATCCTTCTGACCGGACAG, R- GGCTACTGGGGATAAGGGTTG; Roseburia faecis F- 
CGCAACCCCTGTCCTTAGTAG, R- AGATTTGCTCGGCCTCACG. All primers were synthesized by Integrated DNA Technologies (Coralville, IA). Real-time PCR reactions were prepared in a $10 \mu \mathrm{L}$ volume containing 5 $\mu \mathrm{L} 2 x$ SYBR Green, $3 \mu \mathrm{L}$ nuclease free water, $1 \mu \mathrm{L}$ primer mix (a mixture of forward and reverse primer of 5 $\mu \mathrm{M}$ each), and $1 \mu \mathrm{L}$ DNA template. Thermocycling conditions included: (i) an initial denaturation step of 5 min at $95^{\circ} \mathrm{C}$; (ii) 40 cycles of $20 \mathrm{~s}$ at $95^{\circ} \mathrm{C}, 25 \mathrm{~s}$ at annealing temperature $\left(61^{\circ} \mathrm{C}\right.$ for $R$. faecis and $R$. inulinivorans specific primers; $63^{\circ} \mathrm{C}$ for $R$. intestinalis and $R$. homonis specific primers), and $30 \mathrm{~s}$ at $72^{\circ} \mathrm{C}$; (iii) one cycle of $15 \mathrm{~s}$ at $95^{\circ} \mathrm{C}$; (iv) one cycle of $30 \mathrm{~s}$ at $60^{\circ} \mathrm{C}$; (v) one 20 -min interval to generate a melting curve. The cycle threshold of each sample was then compared to a standard curve made by diluting genomic DNA from type strain Roseburia intestinalis DSM 14610, Roseburia inulinivorans DSM 16841, Roseburia hominis DSM 16839 and Roseburia faecis DSM 16840.

\section{SCFA analyses}

SCFA (acetate, propionate, and butyrate) and branched chain fatty acids (BCFA; iso-butyrate and isovalerate) from fermentation samples were analyzed by gas chromatography as described previously [76].

\section{Statistical analysis}

All analyses were performed using $\mathrm{R}$ and Rstudio [86, 87]. Bacterial community diversity was assessed by a-diversity and $\beta$-diversity. a-diversity metrics, including Shannon index, bacterial richness (total ASV numbers) and Pielou's Evenness and $\beta$-diversity in Bray-Curtis distance, were calculated using rarefied ASV data with the phyloseq and vegan packages $[88,89]$. Differences in a-diversity were compared by paired Wilcoxon tests. Differences in the microbiome communities were compared by PERMANOVA using the Adonis function in vegan. In the complementation study, differences in Bray-Curtis distance between different groups to waxy sorghum groups were compared by Kruskal-Wallis test followed by post hoc pairwise multiple comparisons using Dunn's Test. Differential abundance analysis was also performed using LEfSe (Linear discriminant analysis Effect Size) [90]. Bacterial genera and Roseburia species were compared by paired-Wilcoxon test for two groups or Kruskal-Wallis test followed by Dunn's Test for more than two groups.

Differences in in vitro SCFA production and weight gain in mice were compared by repeated measures ANOVA with FDR corrections. Spearman's correlation analysis was performed between fecal SCFA concentrations and the abundances of genera, and between key microbiome features (abundances of genera and Roseburia species and SCFA concentrations) and starch concentration in the complementation experiment. Pearson's correlation analysis was performed between the difference in average weight gain between mice fed with waxy versus wild type sorghum and average relative abundance difference of bacterial genera. Data was visualized using different programs and $\mathrm{R}$ packages [91-94].

\section{Declarations}




\section{Ethical Approval and Consent to participate}

All procedures involving human subjects were approved by the Institutional Review Board of the University of Nebraska-Lincoln before initiating the study (20160816311EP). Informed consent was obtained from all subjects prior to fecal collection. The Institutional Animal Care and Use Committee at the University of Nebraska-Lincoln approved all procedures involving animals (protocol 1700).

\section{Consent for publication}

Not applicable.

\section{Availability of data and materials}

The sequence data reported in this paper have been deposited in the NCBI database (PRJNA811321).

\section{Competing interests}

Not applicable.

\section{Funding}

This research was supported by funds from the Jeff and Tricia Raikes Foundation, the Bill and Melinda Gates Foundation, the Don Dillon Foundation to A.K.B., and USDA-ARS project 3042-21220-033-00D. Q.Y. was supported in part by scholarship from the China Scholarship Council and N.K. was supported in part by Foundation for Food and Agriculture Research (FFAR) and the FFAR Fellows Program.

\section{Authors' contributions}

QY: Conceptualization, Methodology, Formal analysis, Investigation, Writing - original draft, Writing review \& editing. MVH: Methodology, Investigation, review \& editing. NK: Methodology. SS: Resources, Methodology, Writing - review \& editing. DR: Conceptualization, Methodology, Resources, review \& editing. AJ: Methodology, Investigation, review \& editing. JS: Methodology, Investigation. KB: Methodology, Investigation. JP: Methodology, Investigation. JT: Resources, Methodology, Investigation, review \& editing. ART: Conceptualization, Methodology, Resources, review \& editing. AB: Conceptualization, Resources, Writing - original draft, Writing - review \& editing, Supervision, Funding acquisition.

\section{Acknowledgements}

This work was completed utilizing the Holland Computing Center of the University of Nebraska which receives support from the Nebraska Research Initiative. We thank the technical staff from the Nebraska Gnotobiotic Mouse Program for outstanding animal husbandry.

\section{Authors' information}


${ }^{1}$ Department of Food Science and Technology, University of Nebraska-Lincoln, Lincoln, Nebraska, USA.

${ }^{2}$ Nebraska Food for Health Center at the University of Nebraska, Lincoln, Nebraska, USA. ${ }^{3}$ Complex Biosystems Graduate Program, University of Nebraska-Lincoln, Lincoln, Nebraska, USA. ${ }^{4}$ Wheat, Sorghum and Forage Research Unit, USDA-Agricultural Research Service, Lincoln, Nebraska, USA. ${ }^{5}$ Department of Agronomy and Horticulture, University of Nebraska-Lincoln, Lincoln, Nebraska, USA.

\section{References}

1. Abbas KA, K. Khalil S, Meor Hussin AS. Modified Starches and Their Usages in Selected Food Products: A Review Study. J Agric Sci. 2010;2. doi:10.5539/jas.v2n2p90.

2. Chiu C, Solarek D. Modification of Starches. In: Starch. Elsevier; 2009. p. 629-55. doi:10.1016/B9780-12-746275-2.00017-3.

3. James MG, Denyer K, Myers AM. Starch synthesis in the cereal endosperm. Curr Opin Plant Biol. 2003;6:215-22. doi:10.1016/S1369-5266(03)00042-6.

4. Schwartz D, Whistler RL. History and Future of Starch. In: Starch. Elsevier; 2009. p. 1-10. doi:10.1016/B978-0-12-746275-2.00001-X.

5. Šárka E, Dvořáček V. New processing and applications of waxy starch (a review). Journal of Food Engineering. 2017;206:77-87.

6. Bortnowska G, Krzemińska N, Mojka K. Effects of waxy maize and potato starches on the stability and physicochemical properties of model sauces prepared with fresh beef meat. Int J Food Sci Technol. 2013;48:2668-75. doi:10.1111/ijfs.12263.

7. Dapcevic-Hadnadjev T, Dokic L, Pojic M, Hadnadjev M, Torbica A, Rakita S. Rheological properties of dough and quality of bread supplemented with emulsifying polysaccharides. Hem Ind. 2014;68:99106. doi:10.2298/HEMIND130124033D.

8. Shure M, Wessler S, Fedoroff N. Molecular identification and isolation of the Waxy locus in maize. Cell. 1983;35:225-33. doi:10.1016/0092-8674(83)90225-8.

9. Pedersen JF, Bean SR, Graybosch RA, Park SH, Tilley M. Characterization of waxy grain sorghum lines in relation to granule-bound starch synthase. Euphytica. 2005;144:151-6. doi:10.1007/s10681005-5298-5.

10. Yerka MK, Toy JJ, Funnell-Harris DL, Sattler SE, Pedersen JF. Registration of N619 to N640 Grain Sorghum Lines with Waxy or Wild-Type Endosperm. J Plant Regist. 2015;9:249-53. doi:10.3198/jpr2014.06.0043crgs.

11. Isshiki M, Morino K, Nakajima M, Okagaki RJ, Wessler SR, Izawa T, et al. A naturally occurring functional allele of the rice waxy locus has a GT to TT mutation at the 5 ' splice site of the first intron. Plant J. 1998;15:133-8. doi:10.1046/j.1365-313X.1998.00189.x.

12. Santra DK, Heyduck RF, Baltensperger DD, Graybosch RA, Nelson LA, Frickel G, et al. Registration of 'Plateau' Waxy (Amylose-Free) Proso Millet. J Plant Regist. 2015;9:41-3. doi:10.3198/jpr2013.11.0067crc. 
13. Graybosch RA, Baenziger PS, Santra DK, Regassa T, Jin Y, Kolmer J, et al. Registration of 'Mattern' Waxy (Amylose-free) Winter Wheat. J Plant Regist. 2014;8:43-8. doi:10.3198/jpr2013.08.0045crc.

14. Yerka MK, Toy JJ, Funnell-Harris DL, Sattler SE, Pedersen JF. Registration of A/BN641 and RN642 waxy Grain Sorghum Genetic Stocks. J Plant Regist. 2015;9:258-61. doi:10.3198/jpr2014.10.0075crgs.

15. Jane J. Current Understanding on Starch Granule Structures. J Appl Glycosci. 2006;53:205-13. doi:10.5458/jag.53.205.

16. Cerqueira FM, Photenhauer AL, Pollet RM, Brown HA, Koropatkin NM. Starch Digestion by Gut Bacteria: Crowdsourcing for Carbs. Trends Microbiol. 2020;28:95-108. doi:10.1016/j.tim.2019.09.004.

17. Sattler SE, Singh J, Haas EJ, Guo L, Sarath G, Pedersen JF. Two distinct waxy alleles impact the granule-bound starch synthase in sorghum. Mol Breed. 2009;24:349-59. doi:10.1007/s11032-0099296-5.

18. Magallanes-Cruz PA, Flores-Silva PC, Bello-Perez LA. Starch Structure Influences Its Digestibility: A Review. J Food Sci. 2017;82:2016-23. doi:10.1111/1750-3841.13809.

19. Morita T, Ito Y, Brown IL, Ando R, Kiriyama S. In Vitro and In Vivo Digestibility of Native Maize Starch Granules Varying in Amylose Contents. J AOAC Int. 2007;90:1628-34. doi:10.1093/jaoac/90.6.1628.

20. Fitzgerald MA, Rahman S, Resurreccion AP, Concepcion J, Daygon VD, Dipti SS, et al. Identification of a Major Genetic Determinant of Glycaemic Index in Rice. Rice. 2011;4:66-74. doi:10.1007/s12284011-9073-z.

21. Frei M, Siddhuraju P, Becker K. Studies on the in vitro starch digestibility and the glycemic index of six different indigenous rice cultivars from the Philippines. Food Chem. 2003;83:395-402.

22. DeMartino P, Cockburn DW. Resistant starch: impact on the gut microbiome and health. Current Opinion in Biotechnology. 2020;61:66-71.

23. Dhital S, Warren FJ, Butterworth PJ, Ellis PR, Gidley MJ. Mechanisms of starch digestion by aamylase-Structural basis for kinetic properties. Crit Rev Food Sci Nutr. 2017;57:875-92. doi:10.1080/10408398.2014.922043.

24. Marlatt KL, White UA, Beyl RA, Peterson CM, Martin CK, Marco ML, et al. Role of resistant starch on diabetes risk factors in people with prediabetes: Design, conduct, and baseline results of the STARCH trial. Contemp Clin Trials. 2018;65:99-108. doi:10.1016/j.cct.2017.12.005.

25. Sandberg JC, Björck IME, Nilsson AC. Effects of whole grain rye, with and without resistant starch type 2 supplementation, on glucose tolerance, gut hormones, inflammation and appetite regulation in an 11-14.5 hour perspective; a randomized controlled study in healthy subjects. Nutr J. 2017;16:111. doi:10.1186/s12937-017-0246-5.

26. Yuan HC, Meng Y, Bai H, Shen DQ, Wan BC, Chen LY. Meta-analysis indicates that resistant starch lowers serum total cholesterol and low-density cholesterol. Nutrition Research. 2018;54:1-11. doi:10.1016/j.nutres.2018.02.008. 
27. Esgalhado M, Kemp JA, Azevedo R, Paiva BR, Stockler-Pinto MB, Dolenga CJ, et al. Could resistant starch supplementation improve inflammatory and oxidative stress biomarkers and uremic toxins levels in hemodialysis patients? A pilot randomized controlled trial. Food Funct. 2018;9:6508-16. doi:10.1039/c8fo01876f.

28. Birt DF, Boylston T, Hendrich S, Jane JL, Hollis J, Li L, et al. Resistant starch: Promise for improving human health. Advances in Nutrition. 2013;4:587-601. doi:10.3945/an.113.004325.

29. Wang S, Wang J, Yu J, Wang S. A comparative study of annealing of waxy, normal and high-amylose maize starches: The role of amylose molecules. Food Chem. 2014;164:332-8.

30. Bindels LB, Segura Munoz RR, Gomes-Neto JC, Mutemberezi V, Martínez I, Salazar N, et al. Resistant starch can improve insulin sensitivity independently of the gut microbiota. Microbiome. 2017;5:1-16. doi:10.1186/s40168-017-0230-5.

31. Tan J, McKenzie C, Potamitis M, Thorburn AN, Mackay CR, Macia L. The Role of Short-Chain Fatty Acids in Health and Disease. In: Advances in Immunology. Academic Press; 2014. p. 91-119. doi:10.1016/B978-0-12-800100-4.00003-9.

32. Venegas DP, De La Fuente MK, Landskron G, González MJ, Quera R, Dijkstra G, et al. Short chain fatty acids (SCFAs)mediated gut epithelial and immune regulation and its relevance for inflammatory bowel diseases. Frontiers in Immunology. 2019;10 MAR:277. doi:10.3389/fimmu.2019.00277.

33. Slavin J. Fiber and prebiotics: Mechanisms and health benefits. Nutrients. 2013;5:1417-35. doi:10.3390/nu5041417.

34. den Besten G, van Eunen K, Groen AK, Venema K, Reijngoud D-J, Bakker BM. The role of short-chain fatty acids in the interplay between diet, gut microbiota, and host energy metabolism. J Lipid Res. 2013;54:2325-40. doi:10.1194/jlr.R036012.

35. Naseer B, Naik HR, Hussain SZ, Shikari AB, Noor N. Variability in waxy (Wx) allele, in-vitro starch digestibility, glycemic response and textural behaviour of popular Northern Himalayan rice varieties. Sci Rep. 2021;11. doi:10.1038/s41598-021-91537-0.

36. Boers HM, Seijen Ten Hoorn J, Mela DJ. A systematic review of the influence of rice characteristics and processing methods on postprandial glycaemic and insulinaemic responses. British Journal of Nutrition. 2015;114:1035-45. doi:10.1017/S0007114515001841.

37. Louis P, Flint HJ. Formation of propionate and butyrate by the human colonic microbiota. Environmental Microbiology. 2017;19:29-41. doi:10.1111/1462-2920.13589.

38. Gibbon BC, Wang X, Larkins BA. Altered starch structure is associated with endosperm modification in Quality Protein Maize. Proc Natl Acad Sci U S A. 2003;100:15329-34. doi:10.1073/pnas.2136854100.

39. Smits SA, Marcobal A, Higginbottom S, Sonnenburg JL, Kashyap PC. Individualized Responses of Gut Microbiota to Dietary Intervention Modeled in Humanized Mice. mSystems. 2016;1:98-114. doi:10.1128/msystems.00098-16.

40. Cherbuy C, Bellet D, Robert V, Mayeur C, Schwiertz A, Langella P. Modulation of the caecal gut microbiota of mice by dietary supplement containing resistant starch: Impact is donor-dependent. 
Front Microbiol. 2019;10 JUN:1234. doi:10.3389/fmicb.2019.01234.

41. Deehan EC, Yang C, Perez-Muñoz ME, Nguyen NK, Cheng CC, Triador L, et al. Precision Microbiome Modulation with Discrete Dietary Fiber Structures Directs Short-Chain Fatty Acid Production. Cell Host Microbe. 2020;27:389-404.e6. doi:10.1016/j.chom.2020.01.006.

42. Collins NE, Moran ET, Stilborn HL. Performance of broilers fed normal and waxy corn diets formulated with chick and rooster derived apparent metabolizable energy values for the grains. $J$ Appl Poult Res. 2003;12:196-206.

43. Li H, Gidley MJ, Dhital S. High-Amylose Starches to Bridge the “Fiber Gap": Development, Structure, and Nutritional Functionality. Comprehensive Reviews in Food Science and Food Safety. 2019;18:362-79. doi:10.1111/1541-4337.12416.

44. Juliano BO, Hicks PA. Rice functional properties and rice food products. Food Rev Int. 1996;12:71103. doi:10.1080/87559129609541068.

45. Ketthaisong D, Suriharn B, Tangwongchai R, Lertrat K. Changes in physicochemical properties of waxy corn starches after harvest, and in mechanical properties of fresh cooked kernels during storage. Food Chem. 2014;151:561-7.

46. Tamanai-Shacoori Z, Smida I, Bousarghin L, Loreal O, Meuric V, Fong SB, et al. Roseburia spp.: a marker of health? Future Microbiol. 2017;12:157-70. doi:10.2217/fmb-2016-0130.

47. Kumari R. Fluctuations in butyrate-producing bacteria in ulcerative colitis patients of North India. World J Gastroenterol. 2013;19:3404. doi:10.3748/wjg.v19.i22.3404.

48. Si X, Shang W, Zhou Z, Strappe P, Wang B, Bird A, et al. Gut Microbiome-Induced Shift of Acetate to Butyrate Positively Manages Dysbiosis in High Fat Diet. Mol Nutr Food Res. 2018;62:1700670. doi:10.1002/mnfr.201700670.

49. Rivière A, Selak M, Lantin D, Leroy F, De Vuyst L. Bifidobacteria and butyrate-producing colon bacteria: Importance and strategies for their stimulation in the human gut. Frontiers in Microbiology. 2016;7 JUN:979. doi:10.3389/fmicb.2016.00979.

50. Guo P, Zhang K, Ma X, He P. Clostridium species as probiotics: Potentials and challenges. Journal of Animal Science and Biotechnology. 2020;11:1-10. doi:10.1186/s40104-019-0402-1.

51. Seo B, Jeon K, Moon S, Lee K, Kim WK, Jeong H, et al. Roseburia spp. Abundance Associates with Alcohol Consumption in Humans and Its Administration Ameliorates Alcoholic Fatty Liver in Mice. Cell Host Microbe. 2020;27:25-40.e6.

52. Sheridan PO, Martin JC, Lawley TD, Browne HP, Harris HMB, Bernalier-Donadille A, et al. Polysaccharide utilization loci and nutritional specialization in a dominant group of butyrateproducing human colonic firmicutes. Microb Genomics. 2016;2:1-16. doi:10.1099/mgen.0.000043.

53. Louis P, Flint HJ. Diversity, metabolism and microbial ecology of butyrate-producing bacteria from the human large intestine. FEMS Microbiol Lett. 2009;294:1-8. doi:10.1111/j.15746968.2009.01514.x.

54. Walker AW, Ince J, Duncan SH, Webster LM, Holtrop G, Ze X, et al. Dominant and diet-responsive groups of bacteria within the human colonic microbiota. ISME J. 2011;5:220-30. 
doi:10.1038/ismej.2010.118.

55. Maier T V., Lucio M, Lee LH, Verberkmoes NC, Brislawn CJ, Bernhardt J, et al. Impact of dietary resistant starch on the human gut Microbiome, Metaproteome, and Metabolome. MBio. 2017;8:1343-60. doi:10.1128/mBio.01343-17.

56. Goodman AL, Kallstrom G, Faith JJ, Reyes A, Moore A, Dantas G, et al. Extensive personal human gut microbiota culture collections characterized and manipulated in gnotobiotic mice. Proc Natl Acad Sci U S A. 2011;108:6252-7. doi:10.1073/pnas.1102938108.

57. McNulty NP, Yatsunenko T, Hsiao A, Faith JJ, Muegge BD, Goodman AL, et al. The impact of a consortium of fermented milk strains on the gut microbiome of gnotobiotic mice and monozygotic twins. Sci Transl Med. 2011;3. doi:10.1126/scitransImed.3002701.

58. Faith JJ, McNulty NP, Rey FE, Gordon JI. Predicting a human gut microbiota's response to diet in gnotobiotic mice. Science (80-). 2011;333:101-4. doi:10.1126/science.1206025.

59. Kieffer DA, Piccolo BD, Marco ML, Kim EB, Goodson ML, Keenan MJ, et al. Mice fed a high-fat diet supplemented with resistant starch display marked shifts in the liver metabolome concurrent with altered gut bacteria. J Nutr. 2016;146:2476-90. doi:10.3945/jn.116.238931.

60. Kong F, Hua Y, Zeng B, Ning R, Li Y, Zhao J. Gut microbiota signatures of longevity. Current Biology. 2016;26:R832-3. doi:10.1016/j.cub.2016.08.015.

61. Estaki M, Pither J, Baumeister P, Little JP, Gill SK, Ghosh S, et al. Cardiorespiratory fitness as a predictor of intestinal microbial diversity and distinct metagenomic functions. Microbiome. 2016;4:42. doi:10.1186/s40168-016-0189-7.

62. Zakrzewski M, Simms LA, Brown A, Appleyard M, Irwin J, Waddell N, et al. IL23R -Protective Coding Variant Promotes Beneficial Bacteria and Diversity in the lleal Microbiome in Healthy Individuals Without Inflammatory Bowel Disease. J Crohn's Colitis. 2019;13:451-61. doi:10.1093/eccojcc/jjy 188.

63. Goodrich JK, Waters JL, Poole AC, Sutter JL, Koren O, Blekhman R, et al. Human Genetics Shape the Gut Microbiome. Cell. 2014;159:789-99. doi:10.1016/j.cell.2014.09.053.

64. Vojinovic D, Radjabzadeh D, Kurilshikov A, Amin N, Wijmenga C, Franke L, et al. Relationship between gut microbiota and circulating metabolites in population-based cohorts. Nat Commun. 2019;10:1-7. doi:10.1038/s41467-019-13721-1.

65. Waters JL, Ley RE. The human gut bacteria Christensenellaceae are widespread, heritable, and associated with health. BMC Biol. 2019;17:83. doi:10.1186/s12915-019-0699-4.

66. Ferreira-Halder CV, Faria AV de S, Andrade SS. Action and function of Faecalibacterium prausnitzii in health and disease. Best Pract Res Clin Gastroenterol. 2017;31:643-8.

67. Martín R, Miquel S, Benevides L, Bridonneau C, Robert V, Hudault S, et al. Functional Characterization of Novel Faecalibacterium prausnitzii Strains Isolated from Healthy Volunteers: A Step Forward in the Use of F. prausnitzii as a Next-Generation Probiotic. Front Microbiol. 2017;0 JUN:1226.

68. Sokol H, Pigneur B, Watterlot L, Lakhdari O, Bermúdez-Humarán LG, Gratadoux JJ, et al. Faecalibacterium prausnitzii is an anti-inflammatory commensal bacterium identified by gut 
microbiota analysis of Crohn disease patients. Proc Natl Acad Sci U S A. 2008;105:16731-6. doi:10.1073/pnas.0804812105.

69. Miquel S, Martín R, Rossi O, Bermúdez-Humarán LG, Chatel JM, Sokol H, et al. Faecalibacterium prausnitzii and human intestinal health. Curr Opin Microbiol. 2013;16:255-61.

70. Guzman MK De, Parween S, Butardo VM, Alhambra CM, Anacleto R, Seiler C, et al. Investigating glycemic potential of rice by unraveling compositional variations in mature grain and starch mobilization patterns during seed germination. Sci Rep. 2017;7:1-14. doi:10.1038/s41598-01706026-0.

71. Yang J, Keshavarzian A, Rose DJ. Impact of Dietary Fiber Fermentation from Cereal Grains on Metabolite Production by the Fecal Microbiota from Normal Weight and Obese Individuals. J Med Food. 2013;16:862-7. doi:10.1089/jmf.2012.0292.

72. Reichardt N, Vollmer M, Holtrop G, Farquharson FM, Wefers D, Bunzel M, et al. Specific substratedriven changes in human faecal microbiota composition contrast with functional redundancy in short-chain fatty acid production. ISME J. 2018;12:610-22. doi:10.1038/ismej.2017.196.

73. Tao S, Bai Y, Zhou X, Zhao J, Yang H, Zhang S, et al. In Vitro Fermentation Characteristics for Different Ratios of Soluble to Insoluble Dietary Fiber by Fresh Fecal Microbiota from Growing Pigs. ACS Omega. 2019;4:15158-67. doi:10.1021/acsomega.9b01849.

74. Bai Y, Zhao J, Tao S, Zhou X, Pi Y, Gerrits WJ, et al. Effect of dietary fiber fermentation on short-chain fatty acid production and microbial composition in vitro. J Sci Food Agric. 2020;100:4282-91. doi:10.1002/jsfa.10470.

75. Xie XJ, Liang YTS, Seib PA, Tuinstra MR. Wet-Milling of Grain Sorghum of Varying Seed Size Without Steeping. Starch - Stärke. 2006;58:353-9. doi:10.1002/star.200500486.

76. Yang J, Bindels LB, Segura Munoz RR, Martínez I, Walter J, Ramer-Tait AE, et al. Disparate Metabolic Responses in Mice Fed a High-Fat Diet Supplemented with Maize-Derived Non-Digestible Feruloylated Oligo- and Polysaccharides Are Linked to Changes in the Gut Microbiota. PLoS One. 2016;11:e0146144. doi:10.1371/journal.pone.0146144.

77. Benson AK, David JRD, Gilbreth SE, Smith G, Nietfeldt J, Legge R, et al. Microbial Successions Are Associated with Changes in Chemical Profiles of a Model Refrigerated Fresh Pork Sausage during an 80-Day Shelf Life Study. Appl Environ Microbiol. 2014;80:5178-94. doi:10.1128/AEM.00774-14.

78. Kozich JJ, Westcott SL, Baxter NT, Highlander SK, Schloss PD. Development of a Dual-Index Sequencing Strategy and Curation Pipeline for Analyzing Amplicon Sequence Data on the MiSeq Illumina Sequencing Platform. Appl Environ Microbiol. 2013;79:5112-20. doi:10.1128/AEM.0104313.

79. Bolyen E, Rideout JR, Dillon MR, Bokulich NA, Abnet CC, Al-Ghalith GA, et al. Reproducible, interactive, scalable and extensible microbiome data science using QIIME 2. Nat Biotechnol. 2019;37:852-7. doi:10.1038/s41587-019-0209-9.

80. Callahan BJ, McMurdie PJ, Rosen MJ, Han AW, Johnson AJA, Holmes SP. DADA2: High-resolution sample inference from Illumina amplicon data. Nat Methods. 2016;13:581-3. 
doi:10.1038/nmeth.3869.

81. Pedregosa FABIANPEDREGOSA F, Michel V, Grisel OLIVIERGRISEL O, Blondel M, Prettenhofer P, Weiss R, et al. Scikit-learn: Machine Learning in Python. 2011. http://scikit-learn.sourceforge.net. Accessed 19 May 2021.

82. Douglas GM, Maffei VJ, Zaneveld JR, Yurgel SN, Brown JR, Taylor CM, et al. PICRUSt2 for prediction of metagenome functions. Nat Biotechnol. 2020;38:685-8. doi:10.1038/s41587-020-0548-6.

83. Zhang H, Yohe T, Huang L, Entwistle S, Wu P, Yang Z, et al. DbCAN2: A meta server for automated carbohydrate-active enzyme annotation. Nucleic Acids Res. 2018;46:W95-101. doi:10.1093/nar/gky418.

84. Thomsen MCF, Hasman H, Westh $\mathrm{H}$, Kaya H, Lund O. RUCS: rapid identification of PCR primers for unique core sequences. Bioinformatics. 2017;33:3917-21. doi:10.1093/bioinformatics/btx526.

85. Ye J, Coulouris G, Zaretskaya I, Cutcutache I, Rozen S, Madden TL. Primer-BLAST: a tool to design target-specific primers for polymerase chain reaction. BMC Bioinformatics. 2012;13:134. doi:10.1186/1471-2105-13-134.

86. R Core Team. R: A language and environment for statistical computing. 2021. https://www.rproject.org. Accessed 17 May 2021.

87. RStudio Team. RStudio: Integrated Development Environment for R. http://www.rstudio.com/. Accessed 17 May 2021.

88. Oksanen J, Blanchet FG, Friendly M, Kindt R, Legendre P, Mcglinn D, et al. vegan: Community Ecology Package. 2020. https://cran.r-project.org/package=vegan. Accessed 17 May 2021.

89. McMurdie PJ, Holmes S. phyloseq: An R Package for Reproducible Interactive Analysis and Graphics of Microbiome Census Data. PLoS One. 2013;8:e61217. doi:10.1371/journal.pone.0061217.

90. Segata N, Izard J, Waldron L, Gevers D, Miropolsky L, Garrett WS, et al. Metagenomic biomarker discovery and explanation. Genome Biol. 2011;12:R60. doi:10.1186/gb-2011-12-6-r60.

91. Hadley Wickham. ggplot2: Elegant Graphics for Data Analysis. 2016. https://ggplot2.tidyverse.org. Accessed 17 May 2021.

92. Kassambara A. ggpubr: "ggplot2" Based Publication Ready Plots. 2020. https://cran.rproject.org/package=ggpubr. Accessed 17 May 2021.

93. Gu Z, Eils R, Schlesner M. Complex heatmaps reveal patterns and correlations in multidimensional genomic data. Bioinformatics. 2016;32:2847-9. doi:10.1093/bioinformatics/btw313.

94. Letunic I, Bork P. Interactive Tree Of Life (iTOL) v5: an online tool for phylogenetic tree display and annotation. Nucleic Acids Res. 2021;49:W293-6. doi:10.1093/nar/gkab301.

95. GRIN. https://www.ars-grin.gov/Pages/Collections. Accessed 26 May 2021.

\section{Tables}

Table 1. Information for the grain lines used in this study. 


\begin{tabular}{|c|c|c|c|c|c|}
\hline Commodity & Line & $\begin{array}{l}\text { Starch } \\
\text { type }\end{array}$ & $\begin{array}{l}\text { Amylose } \\
\text { content \% } \\
\text { (w/w) }\end{array}$ & $\begin{array}{l}\text { Residual Starch } \\
\text { Content } \%(w / w)\end{array}$ & Source \\
\hline \multirow[t]{12}{*}{ Sorghum } & N619 & Waxy & 1.02 & 0.10 & \multirow{8}{*}{$\begin{array}{l}\text { [10], provided by Scott } \\
\text { Sattler }\end{array}$} \\
\hline & N620 & $\begin{array}{l}\text { Wild } \\
\text { Type }\end{array}$ & 9.80 & 0.21 & \\
\hline & N621 & Waxy & 0.83 & 0.07 & \\
\hline & N622 & $\begin{array}{l}\text { Wild } \\
\text { Type }\end{array}$ & 11.30 & 0.35 & \\
\hline & N625 & Waxy & 0.89 & 0.08 & \\
\hline & N626 & $\begin{array}{l}\text { Wild } \\
\text { Type }\end{array}$ & 10.55 & 0.25 & \\
\hline & N639 & Waxy & 1.04 & 0.07 & \\
\hline & N640 & $\begin{array}{l}\text { Wild } \\
\text { Type }\end{array}$ & 12.58 & 0.27 & \\
\hline & RN642 & Waxy & 0.89 & 0.07 & \multirow{4}{*}{$\begin{array}{l}\text { [14], provided by Scott } \\
\text { Sattler }\end{array}$} \\
\hline & Tx430 & $\begin{array}{l}\text { Wild } \\
\text { Type }\end{array}$ & 11.73 & 0.34 & \\
\hline & BN461 & Waxy & 0.79 & 0.08 & \\
\hline & Wheatland & $\begin{array}{l}\text { Wild } \\
\text { Type }\end{array}$ & 13.27 & 0.40 & \\
\hline \multirow[t]{2}{*}{ Rice } & $\begin{array}{l}\text { Japanese } \\
\text { Sweet Rice }\end{array}$ & Waxy & 0.72 & 0.05 & \multirow[t]{2}{*}{ local grocery store } \\
\hline & $\begin{array}{l}\text { Jasmine } \\
\text { Rice }\end{array}$ & $\begin{array}{l}\text { Wild } \\
\text { Type }\end{array}$ & 7.43 & 0.11 & \\
\hline \multirow[t]{2}{*}{ Wheat } & $\begin{array}{l}\text { Waxy } \\
\text { Wheat }\end{array}$ & Waxy & 0.78 & 0.09 & \multirow[t]{2}{*}{$\begin{array}{l}\text { [13], from UNL wheat } \\
\text { quality lab }\end{array}$} \\
\hline & $\begin{array}{l}\text { Non-Waxy } \\
\text { Wheat }\end{array}$ & $\begin{array}{l}\text { Wild } \\
\text { Type }\end{array}$ & 11.99 & 0.81 & \\
\hline \multirow[t]{2}{*}{ Millet } & Plateau & Waxy & 0.61 & 0.08 & \multirow[t]{2}{*}{$\begin{array}{l}\text { [12], provided by Ismail } \\
\text { Dweikat }\end{array}$} \\
\hline & Hunksman & $\begin{array}{l}\text { Wild } \\
\text { Type }\end{array}$ & 12.13 & 0.54 & \\
\hline Maize & W64A waxy & Waxy & 0.81 & 0.07 & $\begin{array}{l}\text { Ordered from Genetic } \\
\text { Resource Collections, [95] }\end{array}$ \\
\hline
\end{tabular}




\begin{tabular}{llll} 
W64A WT & $\begin{array}{l}\text { Wild } \\
\text { Type }\end{array}$ & 9.74 & 0.47 \\
\hline K55 waxy & Waxy & 0.69 & 0.06 \\
\hline K55 WT & $\begin{array}{l}\text { Wild } \\
\text { Type }\end{array}$ & 11.43 & 0.35
\end{tabular}

\section{Figures}
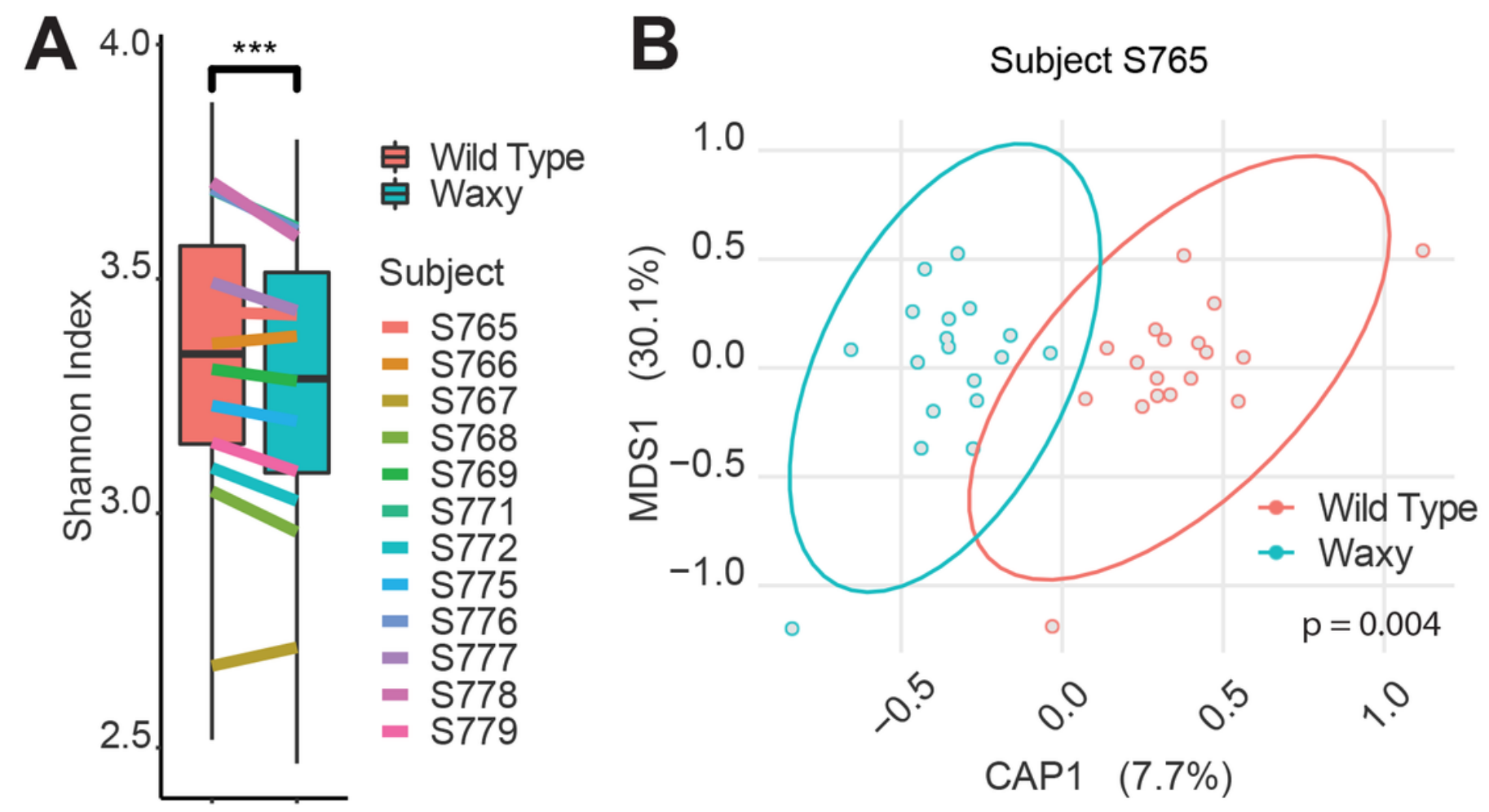

\section{Figure 1}

Effects of waxy sorghum on fecal fermentation bacterial diversity. (A) Box plots of Shannon index for the microbiomes from each subject after fermentation between wild type and waxy sorghum. Lines with different colors represent the average Shannon index of different subjects; Paired Wilcoxon test, $p<0.001$ : ***. (B) Canonical analysis of principal coordinates (CAP) plot based on Bray-Curtis distance, showing the overall microbiome composition difference between waxy and wild type sorghum in subject $\mathrm{S765}$ ( $\mathrm{p}$ value was calculated using PERMANOVA). 


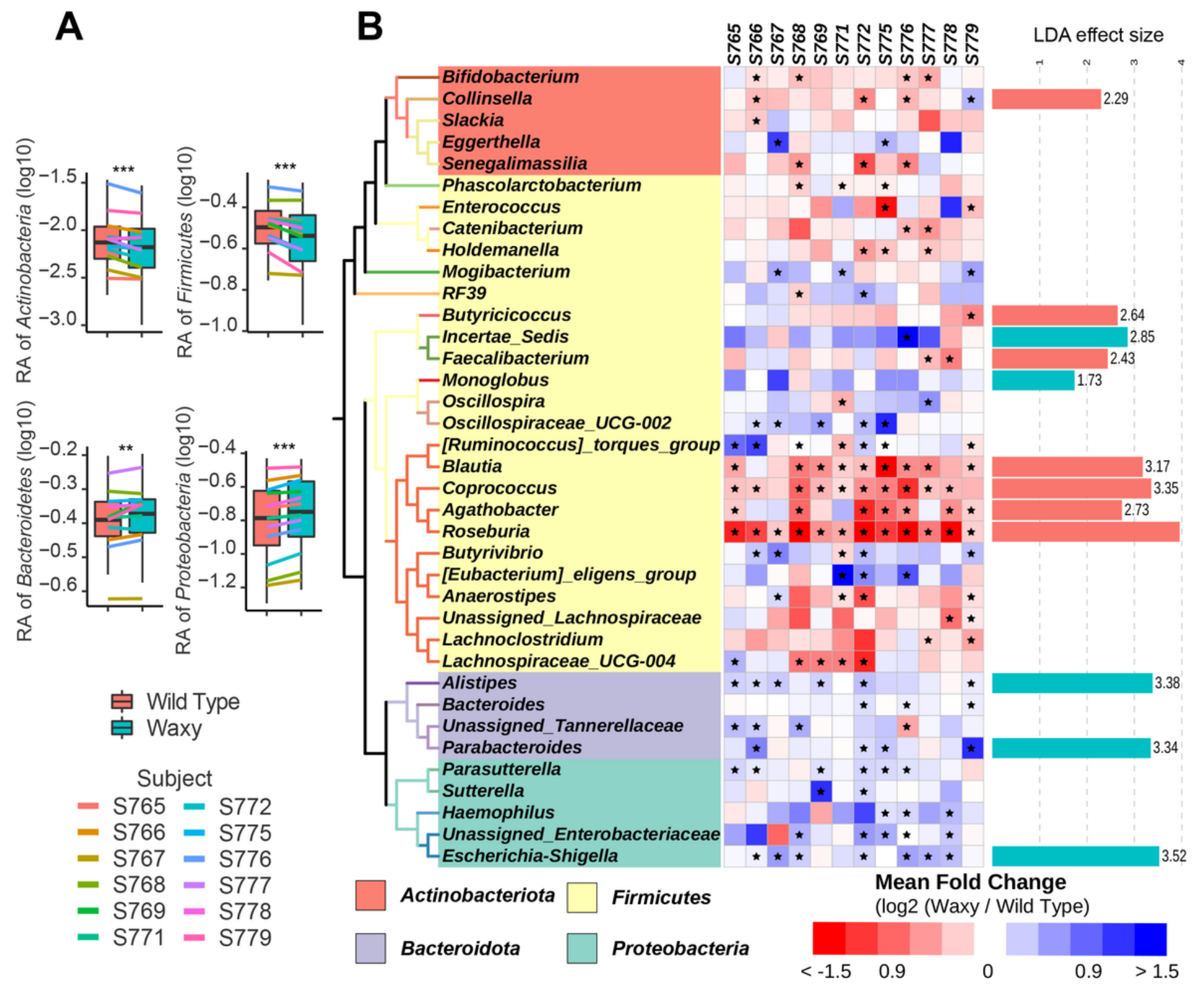

Figure 2

Shifts in the abundance of bacterial taxa between waxy and wild type sorghum. (A) Box plots of the relative abundances of bacterial phyla and one family that showed the most significant difference between waxy and wild type sorghum; lines with different colors represent the average abundance of specific taxa in different subjects (rANOVA followed by FDR correction, $p<0.05$ : $*$; $<0.01$ : $* * ; p<0.001$ : $* \star \star)$. (B) Heatmap of the mean log2-transformed fold change of genera that showed significant effects of wild type sorghum relative to waxy sorghum in each subject. Statistical significance of changes between wild type and waxy sorghum were determined by applying two-way rANOVA (with FDR correction); $q<$ 0.05 considered significant and denoted by asterisk. LEfSe effect size showing genera that were enriched in either wild type or waxy sorghum. 

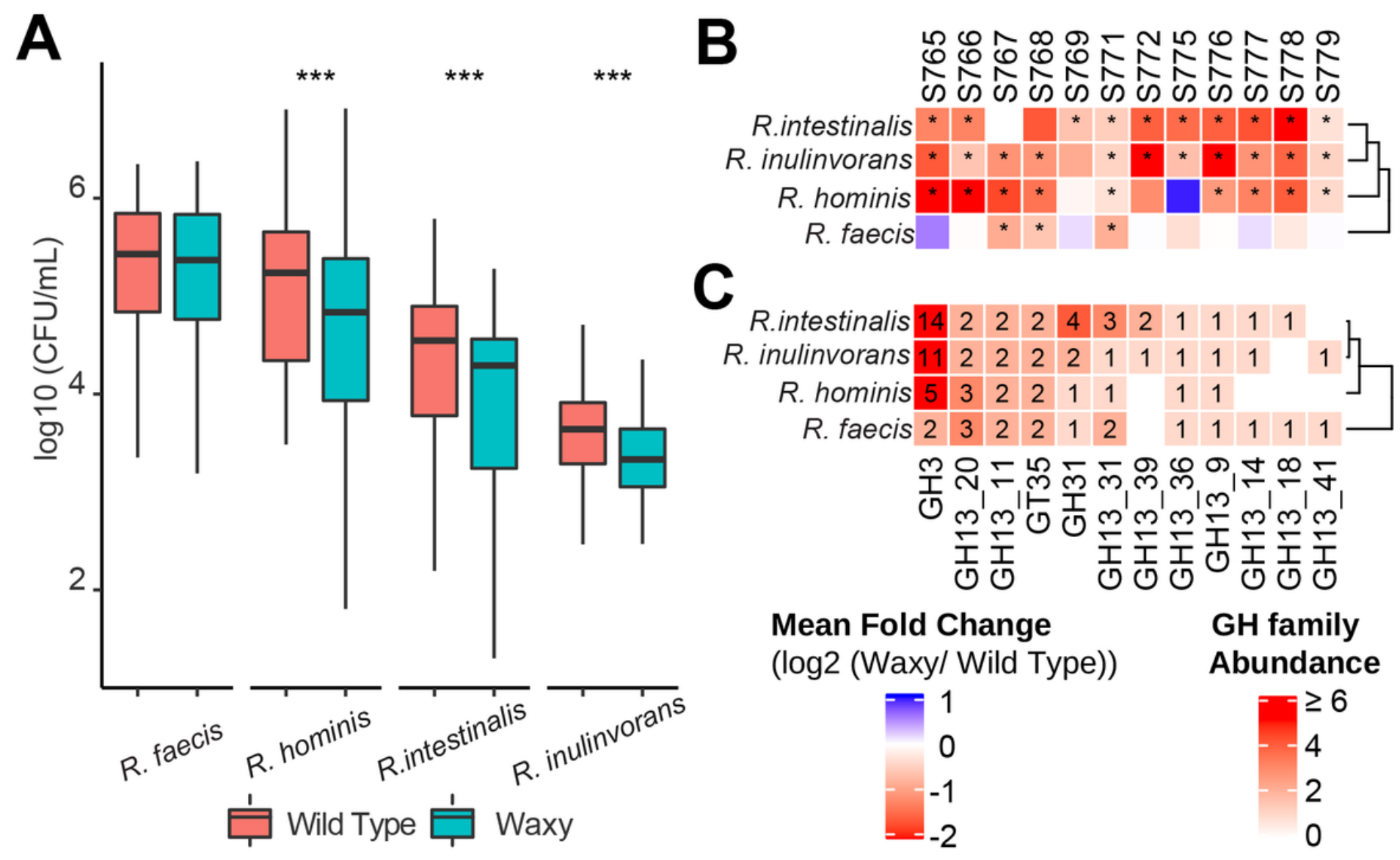

\section{$c$}

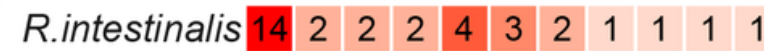

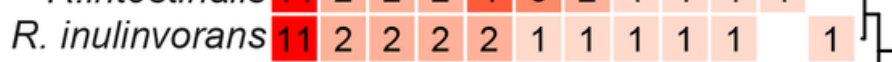

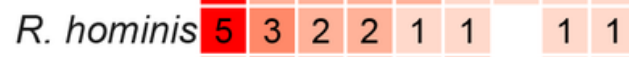

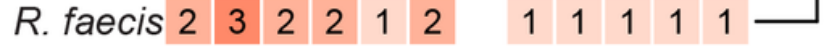
m율

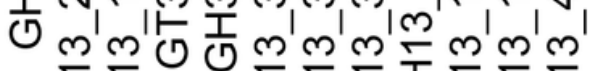

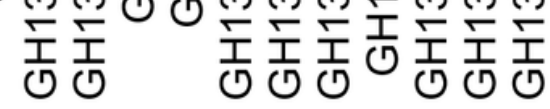

Mean Fold Change (log2 (Waxy/ Wild Type))

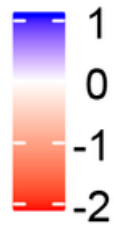

0 1
GH family Abundance

Figure 3

Characterization of Roseburia species between waxy and wild type sorghum fermentations. (A) Box plots of the absolute abundance of different Roseburia species in waxy and wild type sorghum after fermentation (rANOVA followed by FDR correction, $p<0.05$ : $*$; $<0.01$ : $* * ; p<0.001$ : ${ }^{* *}$ ). (B) Heatmap of the mean log2-transformed fold change of different Roseburia species in wild type sorghum relative to waxy sorghum in each subject. Statistical significance of changes between wild type and waxy sorghum were determined using two-way rANOVA (with FDR correction); $p<0.05$ considered significant and denoted with asterisk. (C) Heatmap of starch degradation-associated GH family abundance in different Roseburia species. 

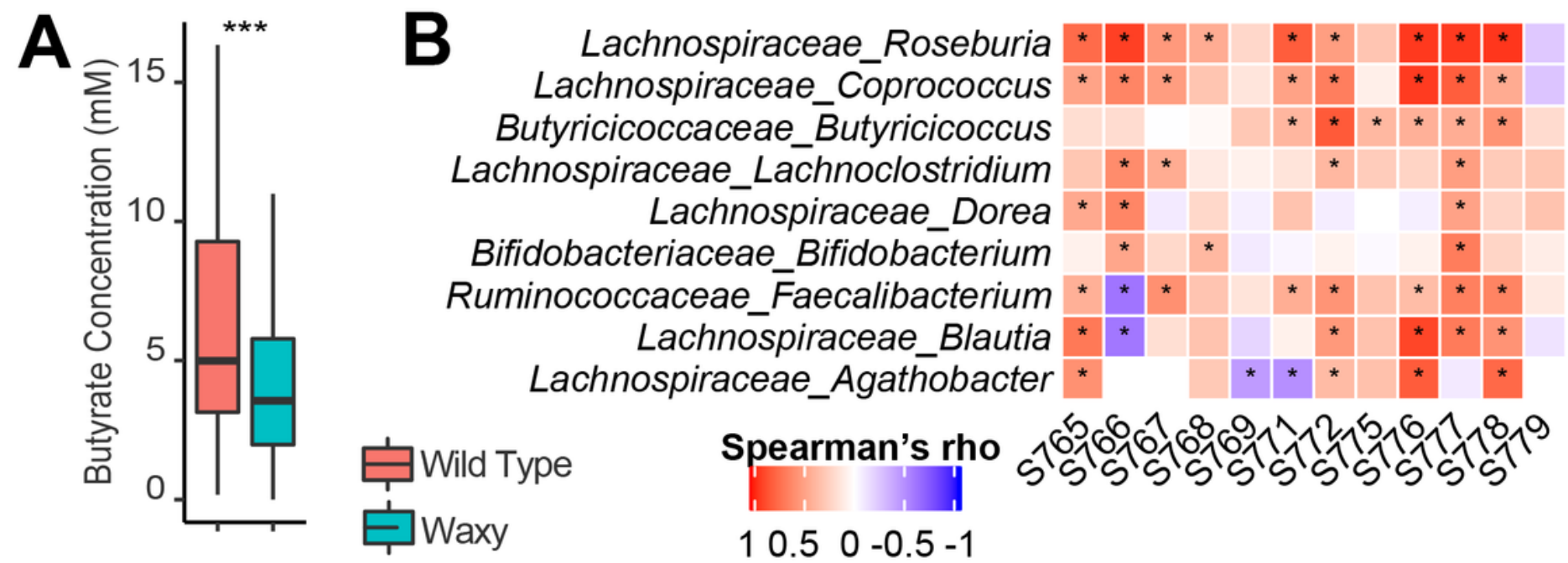

Figure 4

Waxy trait sorghum altered SCFA production. (A) Box plot of concentration of butyrate in waxy and wild type sorghum (rANOVA followed by FDR correction, $p<0.001$ : ***). (B) Heatmap shows the association between butyrate production and relative abundance of specific bacterial genera in each subject using Spearman's correlations; statistical significance of the association was corrected with FDR correction; $\mathrm{p}<$ 0.05 considered significant and denoted by asterisk. 


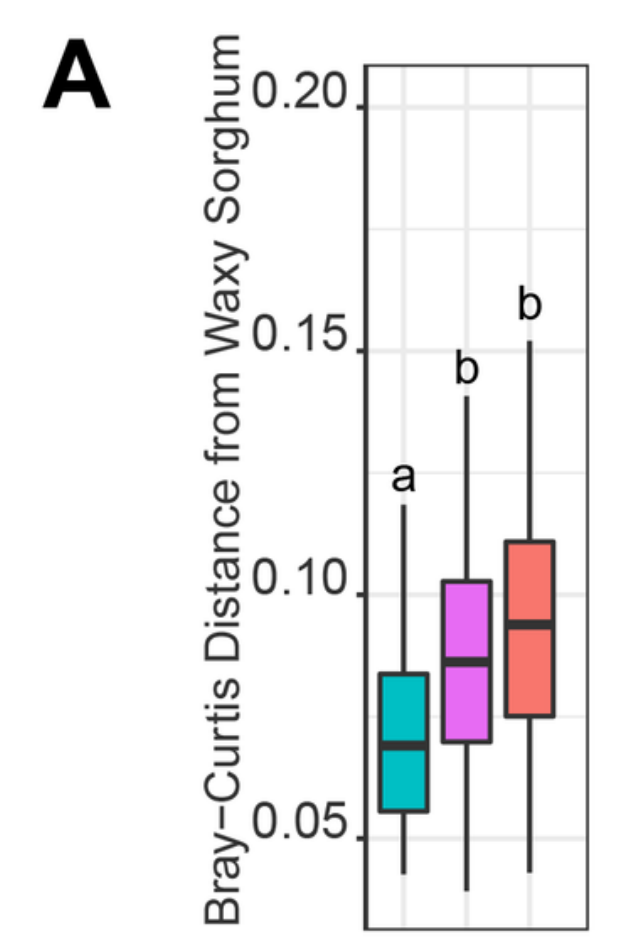

B

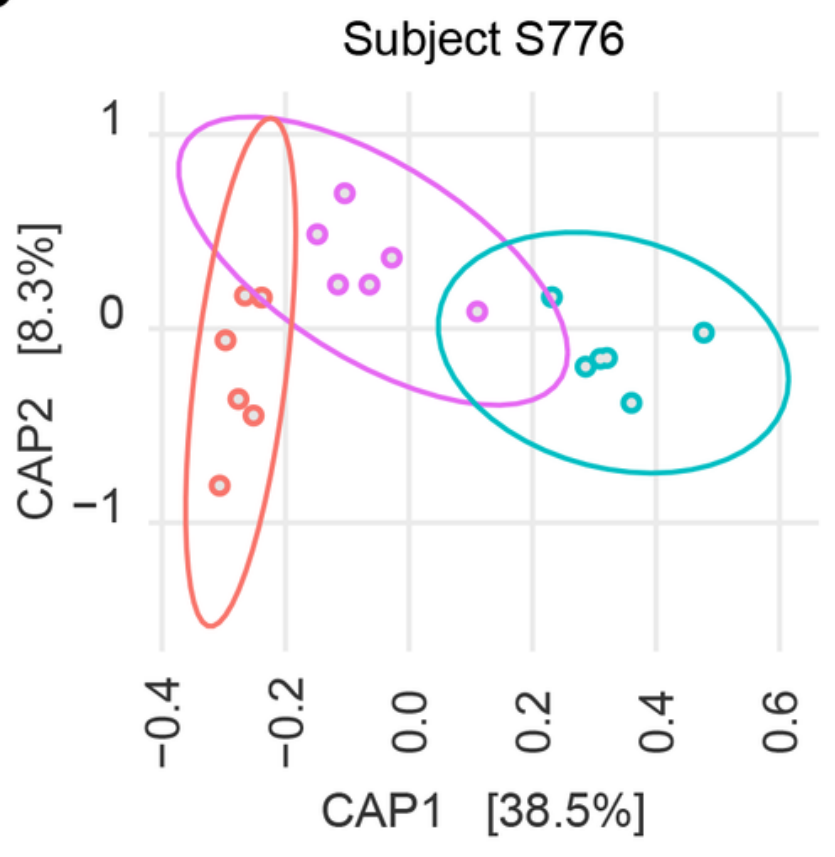

C
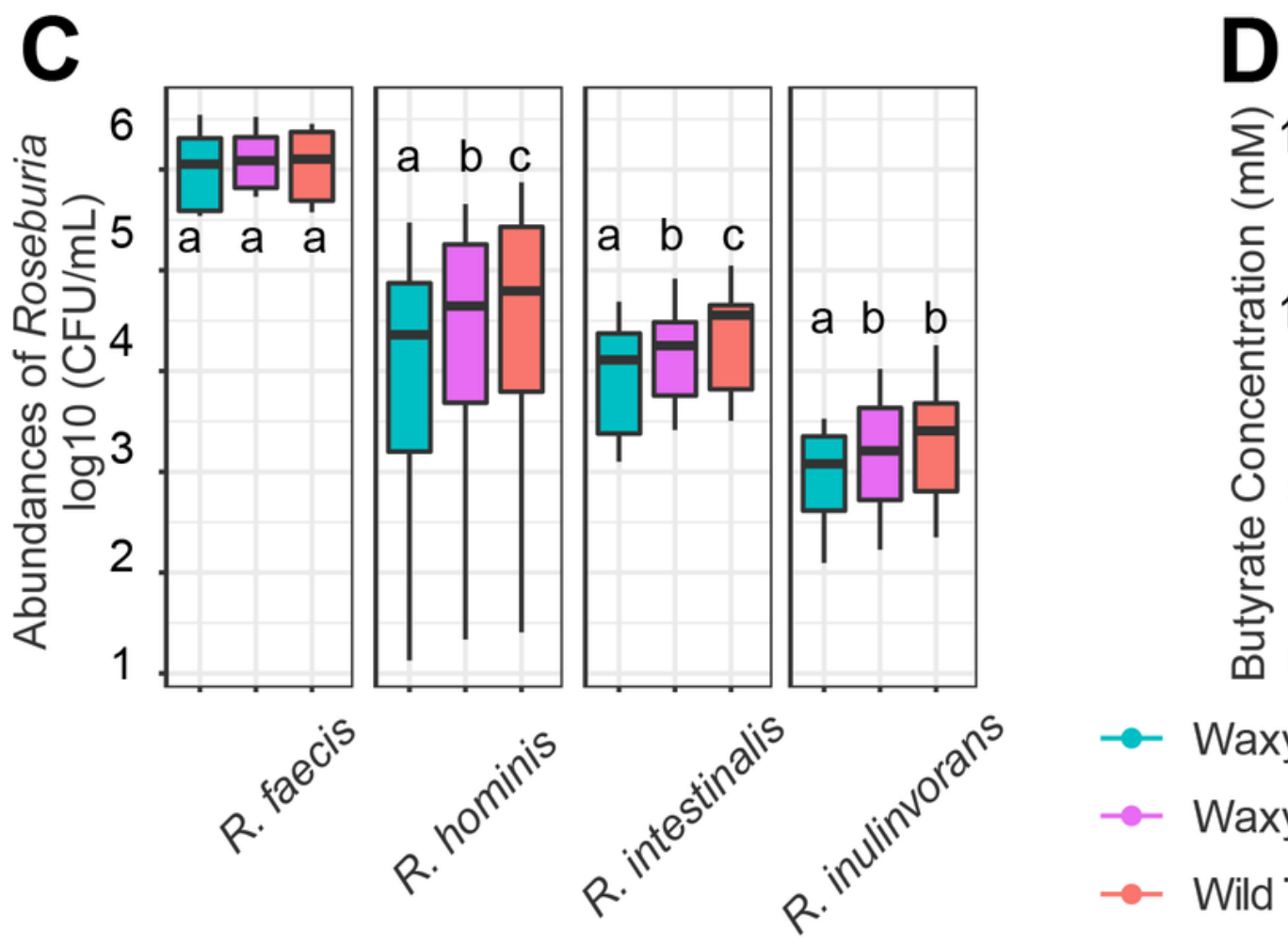

$\multimap$ Waxy

$\rightarrow$ Waxy+Starch

$\rightarrow$ Wild Type

Figure 5

Wild type starch restored microbiome features in waxy sorghum, and wild type starch shows dosedependent effects on bacterial composition and function. (A) Box plot of Bray-Curtis distances between each group to waxy sorghum group; data were analyzed via a Kruskal-Wallis test followed by post hoc pairwise multiple comparisons using Dunn's Test with FDR corrections. (B) Canonical analysis of principal coordinates (CAP) plot based on Bray-Curtis distance showing the overall microbiome 
composition difference across three substrate groups in subject S766 (see Supplemental Figure 3 for additional analyses on Bray-Curtis distance of samples from other subjects). (C) Box plots of the absolute abundance of different Roseburia species in three treatments; data were analyzed via rANOVA followed by FDR correction. (D) Box plots of concentration of butyrate in three threatments; data were analyzed via rANOVA followed by FDR correction. Significant differences are denoted by different letters $(p<0.05)$. (E) Spearman's correlations between starch doses and either the concentrations of butyrate, the abundances of selected genera, or Roseburia species (FDR correction; $p<0.05$ : *).
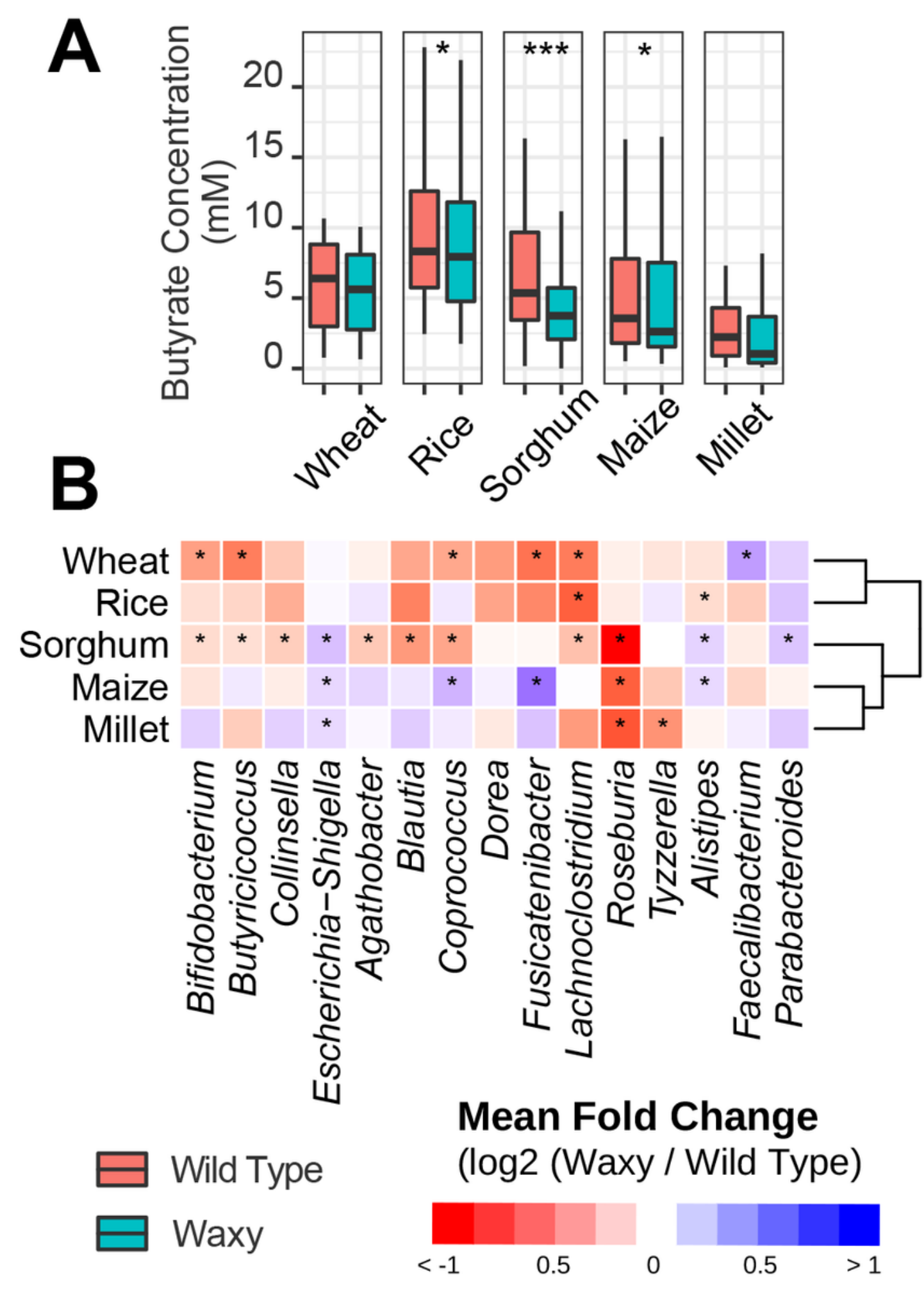
Figure 6

Effect of the waxy trait in different commodities. (A) Box plots of butyrate concentration in different waxy and wild type commodities after in vitro fermentation; data were analyzed via Paired Wilcoxon test, $\mathrm{p}<$ 0.05: $*$; < 0.01: **; $p<0.001$ : ***. (B) Heatmap of the mean log2-transformed fold change of bacterial genera in wild type commodities relative to corresponding waxy commodities; data were analyzed via two-way rANOVA with FDR correction; $\mathrm{p}<0.05$ : *.
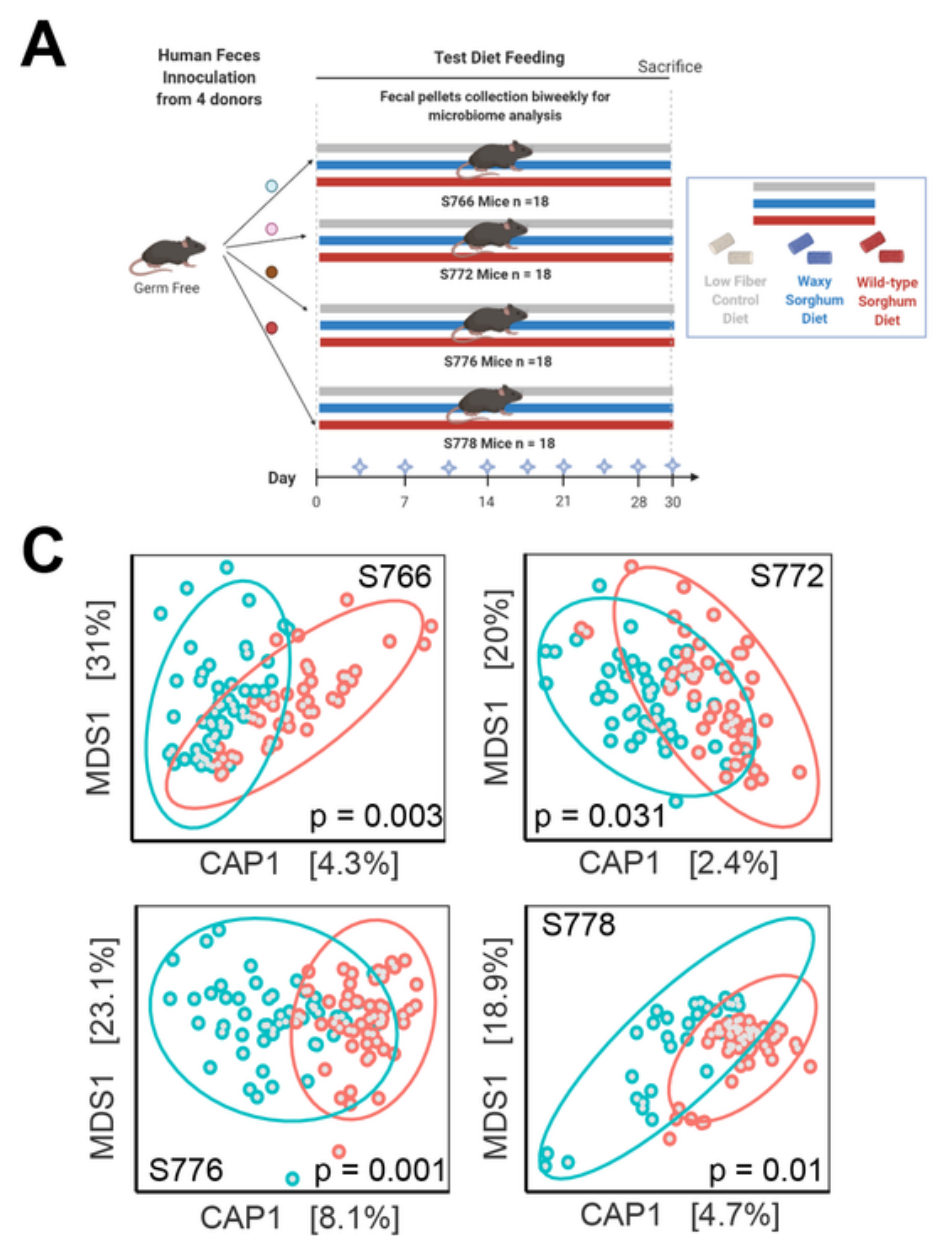

$\mathbf{E}$

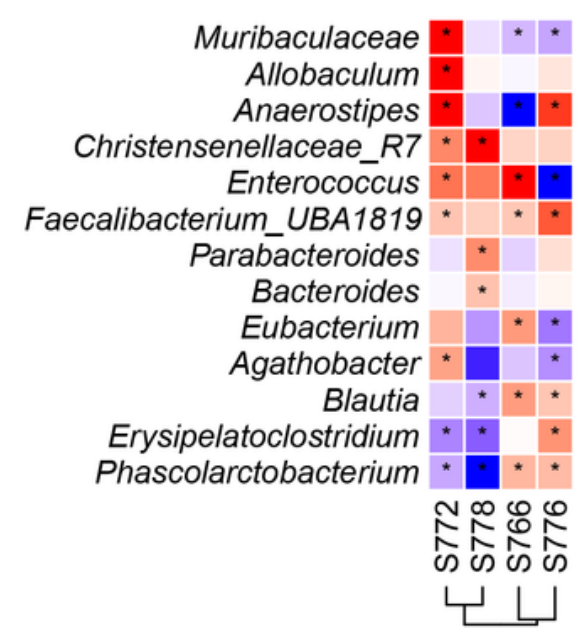

B

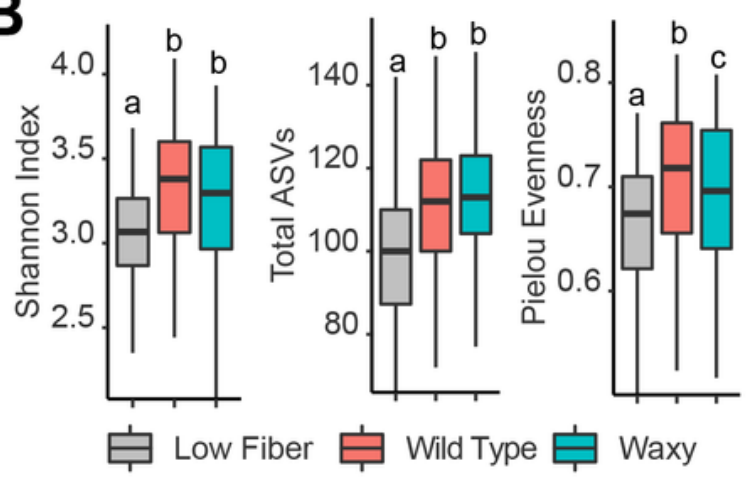

D

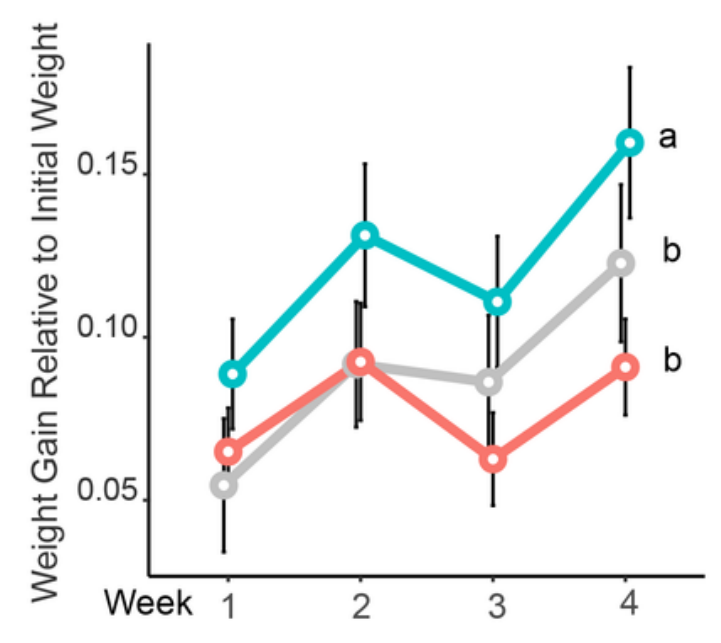

\section{Mean Fold Difference}

(log2 (Waxy/ Wild Type))

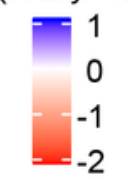




\section{Figure 7}

Effect of waxy sorghum on human microbiome-associated (HMA) mice. (A) Experimental design for mouse feeding study. (B) Box plots of the Shannon index for fecal and cecal microbiomes of mice fed one of three diets; data were analyzed via rANOVA followed by FDR correction. (C) Canonical analysis of principal coordinates (CAP) plot based on Bray-Curtis distance showing the overall microbiome composition differences between mice fed either waxy or wild type sorghum and harboring donor microbiome $S 776$ ( $p$ and $R^{2}$ value were calculated using PERMANOVA, see Supplemental Figure 3 for additional analyses on Bray-Curtis distance of samples from other subjects). (D) Body weight relative to initial weight over time; data are presented as mean \pm SEM; data were analyzed via rANOVA followed by FDR correction. Significant differences are denoted by different letters $(p<0.05)$.

\section{Supplementary Files}

This is a list of supplementary files associated with this preprint. Click to download.

- SupplementalFigure1.pdf

- SupplementalFigure2.pdf

- SupplementalFigure3.pdf

- SupplementalFigure4.pdf

- SupplementaryTable.docx 